\title{
Modal Logics of Reactive Frames
}

\author{
Dov Gabbay \\ King's College London, UK \\ Sérgio Marcelino \\ King's College, UK \\ SQIG - Instituto de Telecomunicações, Portugal * \\ paper: DovPapers/306-TheoryGraphs/306logicsofreactiveframes.tex \\ Partial Draft \\ October 1, 2009
}

\begin{abstract}
A reactive graph generalizes the concept of a graph by making it dynamic, in the sense that the arrows coming out from a point depend on how we got there.

This idea was first applied to Kripke semantics of modal logic in [2]. In this paper we strengthen that unimodal language by adding a second operator. One operator corresponds to the dynamics relation and the other one relates paths with the same endpoint. We explore the expressivity of this interpretation by axiomatizing some natural subclasses of reactive frames.

The main objective of this paper is to present a methodology to study reactive logics using the existent classic techniques.
\end{abstract}

\section{Introduction}

Reactiveness. The reactive idea is very simple. Given a system with states and the possibility of transitions moving between states, we naturally can imagine a path beginning at an initial state and moving along the path following allowed transitions. If our starting point is $s_{0}$, and the path is $s_{0} \ldots s_{n}$, then the system is an ordinary nonreactive system if the options available at $s_{n}$ (i.e. which states $t$ we can go to from $s_{n}$ ) do not depend on the path $s_{0} \ldots s_{n}$ (i.e. do not depend on how we got to $s_{n}$ ). Otherwise if there is such dependence then the system is reactive.

A simple example would be to consider as worlds the configurations on a chess board and the transitions the allowed moves. It is clear that this system is reactive in

${ }^{*}$ This work was partially supported by FCT and EU FEDER, namely via the KLog project PTDC/MAT/68723/2006 of SQIG-IT. Sérgio Marcelino also supported by FCT PhD fellowship SFRH/BD/27938/2006. 
the above sense. To be able to castle one must not have moved either the the king or the rook, it is not enough that they are in their original positions. Moving the king or the rook corresponds to a higher order state transition, changing its nature.

It seems that the simple idea of taking existing systems and making them reactive in certain ways has many new applications. Indeed, there are applications of the reactive ideas in such diverse areas as modal logic, preferential non-monotonic logic, inheritance systems, context free grammars, automata theory, deontic logic and contrary to duty and argumentation and other networks, see papers $[1,2,3,4,6,7,8,9,10,11]$. The list is increasing, see [13].

One can take a reactive system and turn it into an ordinary system by taking the new states as the paths. This is true but from the point of view of applications there is serious loss of information, as the applicability of the reactive system may come from the manner in which the change occurs along the path. In any specific application, the states have meaning, the transitions have meaning and the paths have meaning. Therefore the changes in the system as we go along a path can have very important meaning in the context, which enhances the usability of the model.

Reactive-switch graphs. The ideas and concepts involved are best explained through examples.

Figure 1 presents a simple transition system.

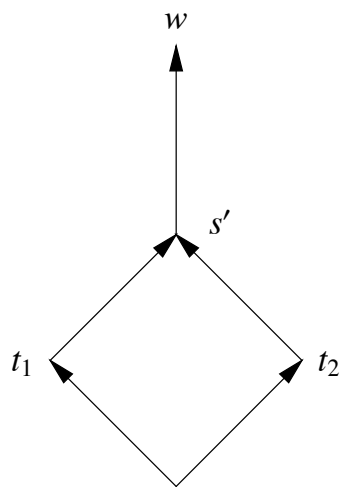

$S$

Figure 1:

We show only the transition options, without details of what causes the transitions. We have the transitions:

$$
\begin{aligned}
& s \rightarrow t_{1} \\
& s \rightarrow t_{2} \\
& t_{1} \rightarrow s^{\prime} \\
& t_{2} \rightarrow s^{\prime} \\
& s^{\prime} \rightarrow w .
\end{aligned}
$$


The system is non-deterministic. Such systems are widely applicable in many areas and their exact meaning depends on the application area in which they are used.

It could be part of an automaton table (where the input letter causing the transition is not shown) or a set of context-free rules, or even part of a Kripke model where $x \rightarrow y$ means $y$ is accessible from $x$.

A reactive graph (or reactive transition system) is a set of sequences of elements of a set, the paths, that contain all the sequences of length one and is closed under prefixes. It is clear it generalizes the static notion of a graph in which every possible extension of a path depends only on its endpoint (see proposition 3.12).

A system becomes reactive when the transition table changes as we move along the graph. We can make the above system reactive by, for example, saying that if we start in $s$, when we reach $s^{\prime}$, it sees $t^{\prime}$ if and only if we pass through $t_{1}$. A natural way of depicting this situation can be found in figure 2 .

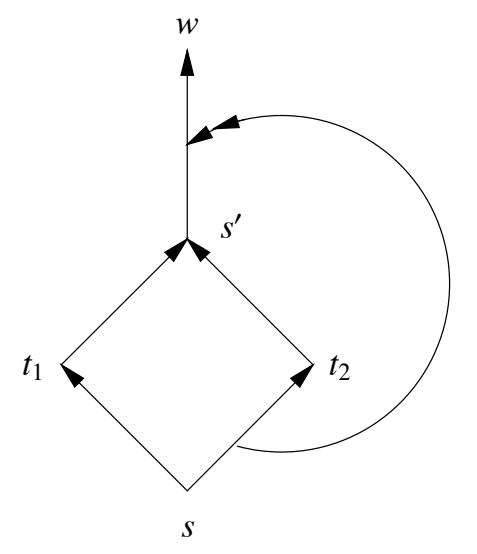

Figure 2:

The double arrow from the edge $s \rightarrow t_{2}$ to the edge $s^{\prime} \rightarrow w$ indicates that if we go from $s$ to $t_{2}$ then the connection $s^{\prime} \rightarrow w$ is no longer available. The double arrow expresses the dependence on the path by codifying the transitions effects on the system configuration. We can make double arrows to act over other double arrows, getting higher and higher levels of dependence.

A switch graph (introduced in [2]) is such a graph enriched with higher order arrows, called the switches. This local way of presenting (generating) the relational dynamics, as it is proved in a paper in preparation, is enough to generate all reactive graphs. There are many possible variations on how to define it. In the mentioned result a switch is an arrow from an edge to another edge or a switch, and it may be a connecting or a disconnecting switch.

Reactive modal logic. As we observed above, a reactive system can be seen in a static point of view by considering their paths as the actual states.

Figure 3 shows the conversion of our example: 


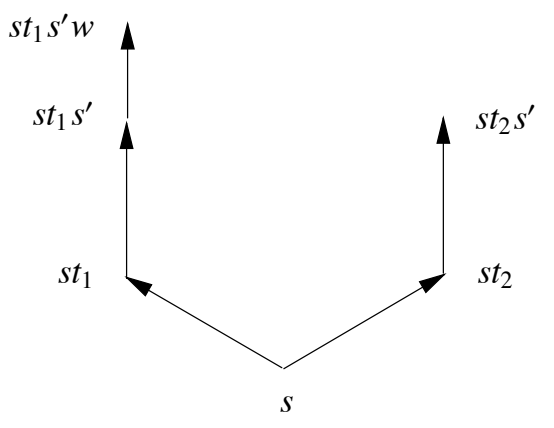

Figure 3:

The points in Figure 3 are the paths. So from the point $s t_{1} s^{\prime}$, there is a transition to $s t_{1} s^{\prime} w$ and from the point $s t_{2} s^{\prime}$ there is no transition to $s t_{2} s^{\prime} w$. That the accessible points vary when in move is already a property of a static graph, what reactivity adds is the possibility of the accessible points from a point to be different depending on how we got there. In this sense the figure 3 is not a proper representation of Figure 2. The two paths $s t_{1} s^{\prime}$ and $s t_{2} s^{\prime}$ share an endpoint (i.e. same state $s^{\prime}$ ) and this can be important. So we need to indicate that by an equivalence relation Figure 4 does that, through the circle around the equivalent points, this is the unfolded version of Figure 2.

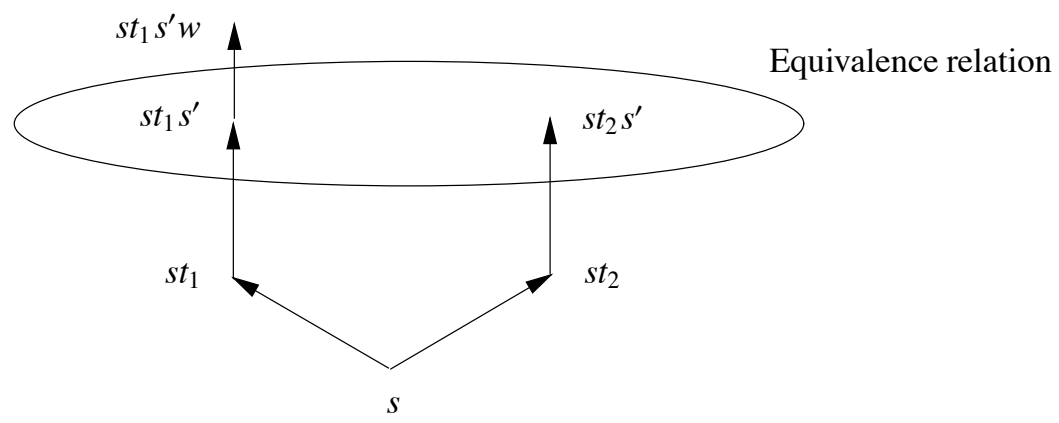

Figure 4:

We will introduce an interpretation of modal logic over reactive graphs (frames), in a way that generalizes the identification between Kripke frames and static graphs, taking in consideration these last remarks.

We were naturally led to the choice of a bimodal language, where $\diamond_{R}$ deals, as usual, with the dynamics and $\diamond_{P}$ with the relation that identifies all the paths with the same endpoint. As we stressed before, reactivity is about the change on the set of accessible points of a given point. $\diamond_{P}$ ranges through the various relational states of each point. As we shall see, many reactive properties can be axiomatised by the interaction between $\diamond_{R}$ and $\diamond_{P}$.

Like the accessibility relation, we let the value of (part of) the variables evolve 
while we go trough the graph. The subset $X$ of variables which we consider fixed, possibly all or none of them, will be a parameter of our logics. So the value of the variables in $X$ depends only on the position in the graph, i.e. the path endpoint. The inclusion of this features in the models gives the results more generality, more possibility of application and allows us to understand better the influence of each component of the generalization in the results.

We can now ask the obvious questions

- what axioms on $\square_{P}, \square_{R}$ correspond to this semantics?

- if we add the reflexivity or transitivity or other obvious axioms on $\square_{R}$, what are the corresponding conditions on the models?

- which other properties can we express with this language?

As an answer we present a procedure to prove that, given a logic and some reactive properties, the first axiomatises the second. While soundness is proved directly, to prove completeness we will use the static view on reactivity by unfolding the models. This allows us to use the canonical model theorem and other classic techniques. When we unfold the notion of a reactive model we obtain a classic bimodal Kripke model, $(W, R, P, V)$ (let $R$ be the dynamics and $P$ the equivalence relation relating the paths with the same end point), satisfying three additional properties:

- there is a family $I \subseteq W$ picking one element on each $P$-equivalence class that $R$-generates all graph;

- $x R y \& x R z \& y P z$ imply that $y \neq z$;

- the worlds related by $P$ satisfy the same variables in $X$.

A shattered frame is a frame satisfying the first property and if it satisfies also the second we say it is a coherently shattered (cs) frame. The completeness proofs are done in two steps:

- we use the canonical model theorem to obtain completeness to a certain subclass of shattered frames corresponding to the reactive one (usually the first-order correspondent of the added axiom)

- we find a truth preserving model transformation that given shattered frame gives a $c s$-frame with that property.

The procedure proves successful in many cases (see in the end of the paper table 1) but we present also its limitations in the form of an open problem. We hope the techniques prove itself useful to characterize logics, possibly in extended languages, expressing reactivity properties coming from research areas where reactivity is being applied to, or to properties suggested by the switch graph research. 


\section{Reactive models}

Definition 2.1 The modal language $\mathcal{L}_{r}$ is defined by

$$
\varphi=p|\neg \varphi| \varphi_{1} \wedge \varphi_{2}\left|\diamond_{R} \varphi\right| \diamond_{P} \varphi
$$

where $p \in \Pi$ is a propositional variable. The other connectives: $\top, \perp, \vee, \rightarrow, \leftrightarrow, \square_{R}$ and $\square_{P}$ are introduced by the usual abbreviations.

Definition 2.2 A path over a set $W$ is any finite sequence of points from $W$. A prefix of a path $w_{0} w_{1} \ldots w_{n}$ is any path of the form $w_{0} w_{1} \ldots w_{k}$ for $k \leq n$. Given a path $\lambda=w_{0} w_{1} \ldots w_{n}$, we let $t(\lambda)=w_{n}$ and $n$ its length.

A reactive frame is a pair $(W, \Delta)$, where $W$ is a non-empty set and $\Delta$ is a set of paths over $W$ that

- contains all one-element paths, i.e., $W$,

- is closed under taking prefixes.

Given $X \subseteq \Pi$, a $X$-reactive model is a triple $\mathcal{M}=(W, \Delta, v)$, where $(W, \Delta)$ is a reactive frame and $v$ is a function $v: \Pi \rightarrow 2^{\Delta}$ such that for $p \in X$ and $\lambda w, \lambda^{\prime} w \in \Delta$ we have $\lambda w \in v(p)$ iff $\lambda^{\prime} w \in v(p)$. X corresponds to the subset of variables that we are fixed while we move, i.e., which value is determined by the current world.

Given a $X$-reactive model $\mathcal{M}$, for every $\lambda \in \Delta$ and every $\mathcal{L}_{r}$-formula $\varphi$, we define the notion ' $\varphi$ is true at $\lambda$ in $\mathcal{M}\left(\mathcal{M}, \lambda \vDash_{X} \varphi\right)$ ' inductively as follows:

- $\mathcal{M}, \lambda \vDash_{X} p$ iff $\lambda \in v(p)$ for variables $p$,

- $\mathcal{M}, \lambda \vDash_{X} \neg \varphi$ iff $\mathcal{M}, \lambda \vDash_{X} \varphi$,

- $\mathcal{M}, \lambda \vDash_{X} \varphi_{1} \wedge \varphi_{2}$ iff $\mathcal{M}, \lambda \vDash_{X} \varphi_{1}$ and $\mathcal{M}, \lambda \vDash_{X} \varphi_{2}$,

- $\mathcal{M}, \lambda \vDash_{X} \diamond_{R} \varphi$ iff there is $w \in W$ such that $\lambda w \in \Delta$ and $\mathcal{M}, \lambda w \vDash_{X} \varphi$,

- $\mathcal{M}, \lambda \vDash_{X} \diamond_{P} \varphi$ iff there is $\gamma \in \Delta$ such that $t(\gamma)=t(\lambda)$ and $\mathcal{M}, \gamma \vDash_{X} \varphi$.

Notice that if $X=\Pi$, then $v$ can be seen as a function from the $\Pi$ to $2^{W}$ and the first line of the definition of $\vDash_{X}$ can be equivalently replaced by $\mathcal{M}, \lambda \vDash_{\Pi} p$ iff $t(\lambda) \in v(p)$.

We say that $\varphi$ is true in $\mathcal{M}$ iff $\mathcal{M}, \lambda \vDash_{X} \varphi$ for every $\lambda \in \Delta$. We say that $\varphi$ is $X$-valid in a reactive frame if it is true in every $X$-reactive model over it.

When $X=\Pi$ we may omit $X$.

Definition 2.3 Given a reactive frame $F=(W, \Delta)$ we define $\sim_{F} \subseteq \Delta^{2}$ as:

$$
\lambda \sim_{F} \gamma \text { iff } t(\lambda)=t(\gamma) \& \forall \beta \in W^{*} \lambda \beta \in \Delta \leftrightarrow \gamma \beta \in \Delta .
$$

We may omit $F$ when it is clear from the context. We write $[\lambda]$ to refer to the equivalence class of $\lambda \in \Delta$. 
Remark 2.4 We will be only interested in studying properties of X-reactive frames, that is, logics valid in the whole class of $X$-reactive models over reactive frames. Otherwise the notion of $\sim$ could be adapted to the context of $X$-reactive models and many of the following results would be valid in its model version.

Proposition 2.5 Let $(W, \Delta)$ be a reactive frame.

1. If $\lambda \sim \gamma$ and $(W, \Delta, v), \lambda \vDash_{\Pi} \varphi$ then $(W, \Delta, v), \gamma \models_{\Pi} \varphi$.

2. If $\lambda, \gamma \in \Delta$ and $\lambda \nsim \gamma$ then there exists $\varphi$ and $v$ s.t. $(W, \Delta, v), \lambda \vDash_{\Pi} \varphi$ and $(W, \Delta, v), \gamma \models_{\Pi} \neg \varphi$.

\section{Proof.}

1. Let us prove it by induction on the structure of $\varphi$ :

- if $\varphi$ is a variable this is trivial since $t(\lambda)=t(\gamma)$.

- if $\varphi=\neg \psi$ then $\mathcal{M}, \lambda \forall_{\Pi} \psi$ and so by IH and symmetry of $\sim, \mathcal{M}, \gamma \forall_{\Pi} \psi$ thus $\mathcal{M}, \gamma \vDash_{\Pi} \neg \psi=\varphi$. The $\varphi=\varphi_{1} \wedge \varphi_{2}$ case is trivial.

- if $\varphi=\diamond_{R} \psi$ then it exists $w \in W$ s.t. $\lambda w \in \Delta$ and $\mathcal{M}, \lambda w \vDash_{\Pi} \psi$. It is clear that we also have $\lambda w \sim \gamma w \in \Delta$ and so by I.H. $\mathcal{M}, \gamma w \vDash_{\Pi} \psi$ hence $\mathcal{M}, \lambda \vDash_{\Pi} \diamond_{R} \psi=\varphi$.

- the case $\varphi=\diamond_{P} \psi$ also comes from $t(\lambda)=t(\gamma)$.

2. If $t(\lambda)=t(\gamma)$ and exists $\beta=w_{1} \ldots w_{n}$ s.t. we do not have $\lambda \beta \in \Delta$ iff $\gamma \beta \in \Delta$. Without loss of generality let us assume $\lambda \beta \in \Delta$ and $\gamma \beta \notin \Delta$ and pick a valuation $v$ that distinguishes all $w_{i}$, i.e., let $p_{i} \in \Pi$ with $1 \leq i \leq n$ and $\alpha \in v\left(p_{i}\right)$ iff $t(\alpha)=w_{i}$. Let $\varphi=\diamond_{R} \varphi_{\beta}$ where $\varphi_{\beta}$ is defined recursively by:

$$
\begin{aligned}
\varphi_{w_{n}} & =p_{n} \\
\varphi_{w_{i} \lambda} & =p_{i} \wedge \diamond_{R} \varphi_{\lambda} .
\end{aligned}
$$

It is clear that $(W, \Delta, v), \lambda \vDash_{\Pi} \varphi$ but $(W, \Delta, v), \gamma \vDash_{\Pi} \varphi$.

If $t(\lambda) \neq t(\gamma)$ we pick a valuation that distinguishes them.

Definition 2.6 $L_{r, X}$ is the logic of all reactive frames:

$$
L_{r, X}=\{\varphi: \varphi \text { is X-valid in every reactive frame }\} .
$$

\subsection{Reactiveness unfolded}

Definition 2.7 1. A shattered frame is a bimodal frame $(W, R, P)$ such that $P$ is an equivalence relation over $W$. Given a shattered frame, we say $I \subseteq W$ is an initial family if it picks one element from each $P$-class that $R$-generates the whole frame. 
2. A cs-frame (coherently shattered) is a shattered frame $(W, R, P)$ that admits an initial family and that is coherent, i.e., such that for all $w, w^{\prime}, w^{\prime \prime} \in W$, if $w R w^{\prime}$, $w R w^{\prime \prime}$ and $w^{\prime} P w^{\prime \prime}$ then $w^{\prime}=w^{\prime \prime}$.

3. A $X$-shattered model is a Kripke model $\mathcal{M}=(W, R, P, V)$ over a shattered frame $(W, R, P)$ and $V$ is $X$-admissible, i.e., for all $w, w^{\prime} \in W$, if $w P w^{\prime}$ then

$$
w \in V(p) \text { iff } w^{\prime} \in V(p) \text { for all } p \in X .
$$

We say $\varphi$ is $X$-valid in a shattered frame if it is true in every $X$-shattered model over it.

4. An $X$-cs-model is a $X$-shattered model over a cs-frame.

Notice that in order for the restrictions on the valuations in a $\Pi$-shattered model $(W, R, P, V)$ to correspond to the restrictions on a general frame $(W, R, P, A)$, where $A$ is the boolean algebra generated by the equivalence classes of $P, A$ has to be closed under $m_{R}$ (and $m_{P}$ ). As we shall see in proposition 3.12 the $c s$-frames that satisfy this requirement are the ones coming from unfolding static reactive frames. But in this case there is no restriction to deal with!

Definition 2.8 $L_{c s, X}$ is the logic of $c s$-frames:

$$
L_{c s, X}=\{\varphi: \varphi \text { is } X \text {-valid in every cs-frame }\} .
$$

Remark 2.9 It is straightforward to see that every reactive frame $F=(W, \Delta)$ can be regarded as cs-frame $F_{c s}=\left(\Delta, R^{\Delta}, P^{\Delta}\right)$ where $W$ is an initial family and

- $\lambda R^{\Delta} \gamma$ iff there is some $w \in W$ such that $\gamma=\lambda w$,

- $\lambda P^{\Delta} \gamma$ iff $t(\lambda)=t(\gamma)$.

We call it the unfolding of $F$. Furthermore it is easy to see that there is a bijective correspondence between $X$-reactive models $\mathcal{M}=(W, \Delta, v)$ and $X$-cs-models $\mathcal{M}_{c s}=$ $\left(\Delta, R^{\Delta}, P^{\Delta}, v\right)$ that preserves truthness, i.e., for every $\lambda \in \Delta$ and every $\mathcal{L}_{r}$-formula $\varphi$,

$$
\mathcal{M}, \lambda \vDash_{X} \varphi \quad \text { iff } \quad \mathcal{M}_{c s}, \lambda \vDash_{X} \varphi
$$

and so we have $L_{c s, X} \subseteq L_{r, X}$.

The converse is also true.

Proposition 2.10 Let $(W, R, P)$ cs-frame with I as initial family. There is a reactive frame s.t. there is bijective correspondence between $X$-cs-model over the first and $X$ reactive models over the second that preserves truthness.

Proof. We call an $R$-path $\lambda=w_{0} \ldots w_{n}$ an $I$-initial path if $w_{0} \in I$. For every such $I$-initial path $\lambda$, let

$$
l_{I}(\lambda)=i_{0} \ldots i_{n}
$$

for the unique sequence of $i_{j} \in I$ with $w_{j} P i_{j}$ for $1 \leq j \leq n$ (see Def. 2.7). 
Moreover $l_{I}$ is an injective function from the set of all $\mathcal{M}$-initial paths into paths over $I$, that is, if $l_{I}(\lambda)=l_{I}(\gamma)$ then $\lambda=\gamma$. Indeed, as $l$ clearly preserves the length of a path, we can do induction on the length $n$ of $\lambda=w_{0} \ldots w_{n}, \gamma=v_{0} \ldots v_{n}$. If $n=0$ then $w_{0}=l_{I}\left(w_{0}\right)=l_{I}\left(v_{0}\right)=v_{0}$ follows. Now suppose that $l_{I}\left(w_{0} \ldots w_{n} w_{n+1}\right)=$ $l_{I}\left(v_{0} \ldots v_{n} v_{n+1}\right)=i_{1} \ldots i_{n} i_{n+1}$ for some $i_{j} \in I, 1 \leq j \leq n+1$. Then $l_{I}\left(w_{0} \ldots w_{n}\right)=$ $l_{I}\left(v_{0} \ldots v_{n}\right)=i_{1} \ldots i_{n}$ and $w_{n+1} P i_{n+1} P v_{n+1}$. By the IH, we have $w_{j}=v_{j}$ for $1 \leq j \leq n$. Therefore not only $w_{n} R w_{n+1}$, but also $w_{n} R v_{n+1}$. So $w_{n+1}=v_{n+1}$ follows by coherence.

Let $\Delta^{I}=\left\{l_{I}(\lambda): \lambda\right.$ is an $I$-initial path $\},\left(I, \Delta^{I}\right)$ is clearly a reactive frame, we call it a folding of $(W, R, P)$.

Given a $X$-cs-model $\mathcal{M}=(W, R, P, V)$ (with $I$ as an initial family) let $\mathcal{M}^{\prime}=\left(I, \Delta^{I}, v\right)$ with

$$
v(p)=\left\{l_{I}(\lambda) \in \Delta^{I}: t(\lambda) \in V(p)\right\}, \text { for each variable } p .
$$

It is straightforward to see that $\mathcal{M}^{\prime}$ is a $X$-reactive model. We will show that $\mathcal{M} \vDash_{X}$ $\varphi$ iff $\mathcal{M}^{\prime} \vDash_{X} \varphi$.

According to Remark 2.9 we can regard $\mathcal{M}^{\prime}$ as a $X$-cs-model $\mathcal{M}_{c s}^{\prime}$ preserving truthness. Hence, to conclude, it is enough to prove that $\mathcal{M}$ is a bounded morphic image of $\mathcal{M}_{c s}^{\prime}$ :

We define a function $f: \Delta^{I} \rightarrow W$ by taking

$$
f\left(l_{I}(\lambda)\right)=t(\lambda)
$$

which is well defined since $l_{I}$ is injective. We claim that $f$ is a surjective bounded morphism from $\mathcal{M}_{c s}^{\prime}$ onto $\mathcal{M}$ :

- $f$ is surjective since by Def. 2.7(1) we have that $I R$-generates all the frame

- p-morphism in $R$. First, if $l_{I}(\lambda), l_{I}(\lambda) i \in \Delta^{I}$ then there is some $w \in W$ such that $t(\lambda) R w$ and $l_{I}(\lambda) i=l_{I}(\lambda w)$. So $t(\lambda) R w=f\left(l_{I}(\lambda) i\right)$. Second, if $f\left(l_{I}(\lambda)\right)=t(\lambda) R w$ then $l_{I}(\lambda w) \in \Delta^{I}$ and $f\left(l_{I}(\lambda w)\right)=w$.

- p-morphism in $P$. First, if $t\left(l_{I}(\lambda)\right)=t\left(l_{I}(\gamma)\right)$ then $t(\lambda) \operatorname{Pt}(\gamma)$. Second, if $f\left(l_{I}(\lambda)\right)=$ $t(\lambda) P w$ then, by Def. 2.7(1), there is some $\mathcal{M}$-initial path $\gamma$ such that $t(\gamma)=w$. Then $l_{I}(\gamma) \in \Delta^{I}$ and $t\left(l_{I}(\lambda)\right)=t\left(l_{I}(\gamma)\right)$ follows.

- p-morphism in $V: l_{I}(\lambda) \in v$ iff $t(\lambda)=f\left(l_{I}(\lambda)\right) \in V(p)$.

Corollary 2.11 $L_{c s, X}=L_{r, X}$.

Proof. From remark 2.9 we get one direction. For the other, suppose that $\varphi \notin L_{c s, X}$, that is, there is some $X$-cs-model $\mathcal{M}=(W, R, P, V)$ having $I$ as initial family and such that $\mathcal{M} \forall_{X} \varphi . \mathcal{M}^{\prime}$ is a $X$-reactive model and $\mathcal{M}^{\prime} \not \forall \varphi$ follows from the previous proposition.

Given a reactive frame we can obtain a cs-frame by unfolding (and vice-versa by folding) where each $X$-model over the first corresponds to a $X$-model over the second and modal satisfaction is preserved. 
With shattered frames we are back in the classic setting even if, in the case of $X \neq \emptyset$, we are not at the frame level. There are restrictions on the models over the correspondent Kripke frames, the situation is closer to the case of general frames.

Folding and unfolding will be the bridge from reactiveness to this classical setting and will allow us to use some known techniques in the study the axiomatisation of logics over reactive frames. The most immediate consequence of this connection is that the logics formed by formulas valid over reactive frames and cs-frames coincide.

\section{Axiomatizations}

In this section we will deal with axiomatizing logics over some classes of reactive frames.

Definition 3.1 Let $L \cup L^{\prime} \cup\{\varphi\}$ be a set of formulas.

- $L \oplus L^{\prime}$ is the closure by the rules of modus ponens and necessity of the set

$$
\left\{\psi: \psi \text { is a propositional instance of } \varphi \in L \cup L^{\prime}\right\} .
$$

If $L, L^{\prime}$ have only one element we may omit the curly brackets.

- $L+_{X} \varphi$ is the closure by the rules of modus ponens and necessity of the set

$L \cup\{\psi: \psi$ is the result of substituting the variables on $\varphi$ by variables in $X\}$.

Let

$$
L_{X}=K_{R} \oplus S 5_{P}+_{X} p \leftrightarrow \square_{P} p,
$$

meaning that $L_{X}$ is the closure by the rules of modus ponens and necessity (for $\square_{R}$ and $\square_{P}$ ) of the set containing

$$
p \leftrightarrow \square_{P} p
$$

for every propositional variable $p \in X$, the substitution instances of all propositional tautologies and of the following axioms:

1. $\square_{R}(p \rightarrow q) \rightarrow\left(\square_{R} p \rightarrow \square_{R} q\right)$

2. $\square_{P}(p \rightarrow q) \rightarrow\left(\square_{P} p \rightarrow \square_{P} q\right)$

3. $\square_{P} p \rightarrow p$

4. $\square_{P} p \rightarrow \square_{P} \square_{P} p$

5. $p \rightarrow \square_{P} \diamond_{P} p$ 
It is clear that $L_{X} \subseteq L_{c s, X}=L_{r, X}$ since all the $L_{X}$ axioms are sound in respect to shattered frames. Notice that the $\diamond_{R}$ fragment of $L_{X}$ is just $K_{R}$, i.e. $K$. It is obvious that $K \subseteq L_{X}$ and from the fact that every Kripke model is a trivial $X$-reactive model we get the other inclusion.

We want to show that $L_{X}$ is complete with respect to all reactive frames. For that we will prove that $L_{X}$ 's canonical model is a $X$-shattered model and then prove that any such model is the bounded image of a generated subframe of a $X$-cs-frame. Hence concluding that $L_{X}=L_{c s, X}=L_{r, X}$.

If $X \neq \emptyset$ then $L_{X}$ is not closed under structural substitution. One can define the canonical model of a normal logic $L$ the usual way, $\mathcal{M}_{L}=\left(W^{L}, R^{L}, P^{L}, V^{L}\right)$ where:

$$
\begin{aligned}
& W^{L}=\{s: s \text { is } L-M C S\} \\
& s R^{L} t \text { iff }\left\{\varphi: \square_{R} \varphi \in s\right\} \subseteq t \text { iff }\left\{\diamond_{R} \varphi: \varphi \in r\right\} \subseteq s \\
& s P^{L} t \text { iff }\left\{\varphi: \square_{P} \varphi \in s\right\} \subseteq t \text { iff }\left\{\diamond_{P} \varphi: \varphi \in r\right\} \subseteq s \\
& V^{L}(p)=\left\{s \in W^{L_{X}}: p \in s\right\} .
\end{aligned}
$$

and prove the well-known truth lemma about it:

$$
\left\{\varphi: \mathcal{M}_{L_{X}} \vDash \varphi\right\}=L_{X}
$$

even if the logic in question is not closed under the rule of substitution, see e.g. [14].

Proposition 3.2 If a logic L contains $p \leftrightarrow \square_{P} p$ and for $p \in X \subseteq \Pi$ then given $v P^{\mathcal{M}_{L}} w$ we have that:

$$
v \in V^{\mathcal{M}_{L}}(p) \Rightarrow w \in V^{\mathcal{M}_{L}}(p) \text { for all } p \in X \text {. }
$$

Proof. If $s P^{\mathcal{M}_{L}} t$ and $p \in s$ then $\square_{P} p \in s$ and so $p \in t$.

Clearly, if $P^{\mathcal{M}_{L}}$ is a equivalence class then all the worlds related by $P^{\mathcal{M}_{L}}$ satisfy the same variables in $X$.

Corollary 3.3 $\mathcal{M}_{L_{X}}$ is $X$-shattered model and so

$$
\{\varphi: \varphi \text { is } X \text {-valid in every shattered frame }\}=L_{X} .
$$

In other words we have that $L_{X}$ is sound and complete with respect to the class of $X$-models over shattered frames. Next, by showing that is also complete with respect to its subclass of cs-frames we conclude the axioms generating $L_{X}$ axiomatise the minimal logic over reactive frames.

Theorem 3.4 $L_{X}=L_{r, X}$

Proof. Since $L_{r, X}=L_{c s, X}$ it is equivalent to prove that $L_{X}=L_{c s, X}$.

Every $c s$-frame is also a shattered frame, hence we have that $L_{c s, X} \subseteq L_{X}$.

Let $\varphi \notin L_{X}$ then exists some $X$-shattered model $\mathcal{M}=(W, R, P, V)$ that does not satisfy $\varphi$. 
We define $\mathcal{N}=\left(W^{\prime}, R^{\prime}, P^{\prime}, V^{\prime}\right)$, where:

$$
\begin{aligned}
& W^{\prime}=\{(z, y): y P z\} \cup W \times\{*\} \\
& (x, i) R^{\prime}(y, j) \text { iff } i=* \& x=y \text { or } i \neq * \& x R y \& y=j \\
& (x, i) P^{\prime}(y, j) \text { iff } i=j \&(i=* \rightarrow x=y) \\
& (x, i) \in V^{\prime}(p) \text { iff } x \in V(p) .
\end{aligned}
$$

$\mathcal{N}$ is a $X$-cs-model and $\mathcal{M}$ is a bounded morphic image of the submodel generated by $W^{\prime \prime}=W^{\prime}-W \times\{*\}$ (and therefore $\left.\varphi \notin L_{c s, X}\right)$ :

- let $I$ be a any family of elements of $W^{\prime}$ picking one element from each $P$-class. It is clear that $W \times\{*\} \subseteq I$ and so $I$ generates the $\mathcal{N}$.

$V^{\prime}$ is an $X$-admissible valuation: $(x, i) P^{\prime}(y, j)$ imply $i=j$ hence $x P y$ and therefore if $p \in X$,

$$
(x, i) \in V^{\prime}(p) \text { iff } z \in V(p) \text { iff } y \in V(p) \text { iff }(y, j) \in V^{\prime}(p) .
$$

Let us check that it is coherent. Given $(x, i),(y, j),(z, k) \in W^{\prime}$. If $(y, j) P^{\prime}(z, k)$ then $j=k$. Let us assume also $(x, i) R^{\prime}(y, j)$ and $(x, i) R^{\prime}(z, k)$. If $i=*$ then $x=y=z$ and $i \neq *$ then $y=j=k=z$. So $(y, j)=(z, k)$.

- let $f: W^{\prime \prime} \rightarrow W$ be defined by: $f((x, i))=x$.

It is straightforward to see that $f$ is surjective, let us see that it is actually a p-morphism:

- $f$ is a p-morphism in $R$

* $(x, i) R^{\prime}(y, j)$ then $f((x, i))=x R y=f((y, j))$.

* $f((x, i)) R y$ then $(x, i) R^{\prime}(y, y)$.

- $f$ is a p-morphism in $P$

* $(x, i) P^{\prime}(y, j)$ then $i=j$ and so, by construction, we have $f((x, i))=$ $x P y=f((y, j))$.

* $f((x, i)) P y$ then $(x, i) P(y, i)$

- $f$ is a p-morphism in $V$

$(x, y) \in V^{\prime}(p)$ iff $f((x, i))=x \in V^{\prime}(p)$.

To this way of generating a $X$-cs-model from a given $X$-shattered model we will call the blow up trick and it is inspired by the standard extensions used in [15].

If we consider the basic properties in graphs like reflexivity, symmetry or transitivity, we see that there are many ways of generalising it to the reactive graph level. This properties refer to the accessibility of points without referring to the changes in their relational state since it is always the same. In the reactive case this is is not true and 
they may mean different things. Reflexivity can mean for example that we can always access loop without any change on the set of accessible points or we may require anything else. All the mentioned properties have similar variants, they are the generalized notions of the originals.

Just as the (static) graphs properties are studied in classic modal logic, we dedicate the rest of the paper to study the logics of the subclasses of reactive frames satisfying some of these properties.

Our strategy will be the same as we used for the general case. Given a class of reactive frames and set of candidate axioms, we first check if they correspond to the reactive frame property in question (in some cases we only have soundness). Then we prove completeness in steps:

- we establish that the logic they originate is complete with respect to class of shattered frames with a certain property by analysing its canonical model;

- we check that its subclass of cs-frames validates exactly the same formulas by showing the existence of a transformation from shattered frame to cs-frames (using the blow up trick) that preserves the class we are considering and showing the first one is a bounded morphic image of a generated submodel of the second;

- we show that folding these cs-frames gives origin to reactive frames with the required property, thus obtaining the result by applying of proposition 2.10 like in corollary 2.11 .

\subsection{Reflexivity and transitivity}

Now we will study some subclasses of reactive frames obtained by imposing properties that generalize the notions of reflexivity and transitivity in the static case and axiomatise them.

Let us introduce some variations of the blow up trick that will work for all the next cases.

Proposition 3.5 Given $\varphi$ and some $X$-shattered model $\mathcal{M}=(W, R, P, V)$ we define $t_{i}(\mathcal{M})=\left(W_{i}, R_{i}, P_{i}, V_{i}\right)$ for $i=1,2,3,4$ :

1. - $W_{1}=\{(x,(y, 0)),(x,(y, 1)): x P y\} \cup W \times\{*\}$

- $(x, i) R_{1}(y, j)$ iff $i=* \& x=y$ or

$\left(i=\left(x^{\prime}, a\right) \& j=\left(y^{\prime}, b\right) \&\right.$

$\left(b=|a-1| \& y=t^{\prime}\left(x, y^{\prime}\right)\right.$ or $\left.\left.i=j \& y=t^{\prime}(x, x)\right)\right)$

- $(x, i) P_{1}(y, j)$ iff $i=j \&(i=1 \rightarrow x=y)$

- $(z, i) \in V_{1}(p)$ iff $z \in V(p)$.

2. - $W_{2}=\left\{\left(t^{n}(c), n, c, d, i\right): c P d ; i=0,1, * ; n<\omega\right\}$

- $(a, b, c, d, i) R_{2}\left(a^{\prime}, b^{\prime}, c^{\prime}, d^{\prime}, i^{\prime}\right)$ iff $(c, d)=\left(c^{\prime}, d^{\prime}\right) \&$

$\left(i=* \&(a, b)=\left(a^{\prime}, b^{\prime}\right)\right.$ or

$i \neq * \& a R a^{\prime} \&$

$\left(a=t\left(a^{\prime}\right) \& b=b^{\prime}+1 \& i=i^{\prime}\right.$ or

$\left.\left.a^{\prime}=c^{\prime}=d^{\prime} \& b^{\prime}=0 \& i=\left|i^{\prime}-1\right|\right)\right)$ 
- $w=(a, b, c, d, i) P_{2}\left(a^{\prime}, b^{\prime}, c^{\prime}, d^{\prime}, i^{\prime}\right)=w^{\prime}$ iff $(d, i)=\left(d^{\prime}, i^{\prime}\right) \&$

$\left(i=* \rightarrow w=w^{\prime}\right)$

- $(a, b, c, d, i) \in V_{2}(p)$ iff $a \in V(p)$

$\left(t^{0}(w)=w\right)$

3. - $W_{3}=\{((x, y), n),(x, n): x P y, n<\omega\}$

- $(x, i) R_{3}(y, j)$ iff $y=\left(y_{1}, y_{2}\right) \&$

$\left(x \in W \&\left(x=y_{1} \& i=j\right.\right.$ or $\left.j>i \& y_{1}=y_{2}\right)$ or $\left.x=\left(x_{1}, x_{2}\right) \& j>i \& x_{1} R y_{1}=y_{2}\right)$

- $(x, i) P_{3}(y, j)$ iff $i=j \&\left(x \in W \& x=y\right.$ or $\left.x=\left(x_{1}, w\right) \& y=\left(y_{1}, w\right)\right)$

- $(x, i) \in V_{3}(p)$ iff $x \in W \& x \in V(P)$ or $x=\left(x_{1}, x_{2}\right) \& x_{1} \in V(p)$.

4. $\quad W_{4}=W_{3}, P_{4}=P_{3} \& V_{4}=V_{3}$

- $(x, i) R_{4}(y, j)$ iff $y=\left(y_{1}, y_{2}\right) \&$

$\left(x \in W \&\left(x=y_{1} \& i=j\right.\right.$ or $\left.j>i \& y_{1}=y_{2}\right)$ or

$\left.x=\left(x_{1}, x_{2}\right) \& j>i \& t^{\prime}\left(x_{1}, y_{2}\right)=y_{1}\right)$

where $t, t^{\prime}$ are defined by

- $t(w)=v$ be s.t. $v R w P v$ if there is such $v$, otherwise it is undefined,

- $t^{\prime}(w, v)=v^{\prime}$, s.t. $w R v^{\prime} P v$ and $w R v \rightarrow v^{\prime}=v$ if there is such $a v^{\prime}$, and is undefined otherwise.

$t_{i}(\mathcal{M})$ is a $X$-cs-model and $\mathcal{M}$ is a bounded morphic image of a generated submodel of $t_{i}(\mathcal{M})$ for $i=1,2,3,4$.

Remark 3.6 Any of these constructions could have been used in theorem 3.4 instead of $\mathcal{N}$.

\section{Proof.}

1. $t_{1}(\mathcal{M})$ is a $X$-cs-model and $\mathcal{M}$ is a bounded morphic image of the submodel of $t_{1}(\mathcal{M})$ generated by $W_{1}^{\prime}=\{(x,(y, 0)),(x,(y, 1)): x P y\}$ :

- the existence of an initial family and that $V_{1}$ is an $X$-admissible valuation is dealt just like in prop. 3.4.

Let us check that it is coherent. Given $(x, i),(y, j),(z, k) \in W_{1}$. If $(y, j) P_{1}(z, k)$ then $j=k$. Let us assume that we also have $(x, i) R_{1}(y, j)$ and $(x, i) R_{1}(z, k)$. If $i=*$ we get $x=y=z$. If $i=(z, a)$ then $j=(w, b)=k$. If $a=b$ then $y=t(x, x)=z$ and if $a \neq b$ then $y=t(x, w)=z$. In any case $(y, j)=(z, k)$.

- let $f: W_{1}^{\prime} \rightarrow W$ be defined by: $f((x, i))=x$. It is straightforward to see that it is a surjective function, let us see that it is actually a p-morphism:

- $f$ is a p-morphism in $R$

$*\left(x,\left(x^{\prime}, i\right)\right) R_{1}\left(y,\left(y^{\prime}, j\right)\right)$ then or $y=t^{\prime}\left(x, y^{\prime}\right)$ or $y=t^{\prime}(x, x)$. In both cases $f((x, i))=x R y=f((y, j))$, 
* $f((x,(t, a))) R y$ then we have that $t^{\prime}(x, y)=y$, hence $(x,(t, a)) R_{1}(y,(y,|a-1|))$;

- $f$ is a p-morphism in $P$

* $(x, i) P_{1}(y, j)$ then $i=j$ and so $f((x, i))=x P y=f((y, j))$,

* $f((x, i)) P y$ then $(x, i) P_{1}(y, i)$;

- $f$ is a p-morphism in $V$, is trivial since by definition $(x, i) \in V_{1}(p)$ iff $f((x, i))=x \in V$.

2. $t_{2}(\mathcal{M})$ is a $X$-cs-model and $\mathcal{M}$ is a bounded morphic image of the submodel of $t_{2}(\mathcal{M})$ generated by $W_{2}^{\prime}=\left\{\left(t^{n}(c), n, c, d, i\right): c P d ; i=0,1 ; n<\omega\right\}$ :

- it is easy to verify that $V_{2}$ is admissible and that any choice of I, picking one element from each $P$-class and containing $\left\{\left(t^{n}(c), n, c, d, *\right): c P d ; n<\omega\right\}$, works as initial family.

Let us check that it is coherent. Given $w=(a, b, c, d, i), w^{\prime}=\left(a^{\prime}, b^{\prime}, c^{\prime}, d^{\prime}, i^{\prime}\right)$ and $w^{\prime \prime}=\left(a^{\prime \prime}, b^{\prime \prime}, c^{\prime \prime}, d^{\prime \prime}, i^{\prime \prime}\right)$ in $W_{2}$. If $w^{\prime} P^{\prime} w^{\prime \prime}$ then $\left(d^{\prime}, i^{\prime}\right)=\left(d^{\prime \prime}, i^{\prime \prime}\right)$. Let us assume that we also have $w R_{2} w^{\prime}$ and $w R_{2} w^{\prime \prime}$ so $\left(c^{\prime}, d^{\prime}\right)=(c, d)=$ $\left(c^{\prime \prime}, d^{\prime \prime}\right)$. If $i=*$ we get $(a, b)=\left(a^{\prime}, b^{\prime}\right)=\left(a^{\prime \prime}, b^{\prime \prime}\right)$. Otherwise, if either $i=$ $i^{\prime}$ or $i=i^{\prime \prime}$ then $i=i^{\prime}=i^{\prime \prime}$, so $b^{\prime}=b-1=b^{\prime \prime}$ and $a=t^{b^{\prime}}(c)=t^{b^{\prime \prime}}(c)=a^{\prime \prime}$. If $i \neq i^{\prime}$ then $b=b^{\prime}=0, a^{\prime}=c^{\prime}=d^{\prime}=d^{\prime \prime}=c^{\prime \prime}=a^{\prime \prime}$. Hence, in any case, $w^{\prime}=w^{\prime \prime}$.

- let $f: W_{2}^{\prime} \rightarrow W$ be defined by $f((a, b, c, d, i))=a$.

It is straightforward to see that it is a surjective function, let us see that it is actually a p-morphism:

The condition in the valuation is trivial as before;

- $f$ is a p-morphism in $R$

* $w=(a, b, c, d, i) R_{2}\left(a^{\prime}, b^{\prime}, c^{\prime}, d^{\prime}, i^{\prime}\right)=w^{\prime}$ then $f((x, i))=a R b=$ $f((y, j))$,

* $f((a, b, c, d, i)) R y$ then or $(a, b, c, d, i) R_{2}(y, 0, y, y,|i-1|)$;

- $f$ is a p-morphism in $P$

* $w=(a, b, c, d, i) P_{2}\left(a^{\prime}, b^{\prime}, c^{\prime}, d^{\prime}, i^{\prime}\right)=w^{\prime}$ then $a P c P d=d^{\prime} P c^{\prime} P a^{\prime}$. Thus, by transitivity of $P$, we obtain $f(w)=a P a^{\prime}=f\left(w^{\prime}\right)$.

* $f((a, b, c, d, i)) P y$ then $(a, b, c, d, i) P_{2}(y, 0, y, d, i)$.

3. $t_{3}(\mathcal{M})$ is a $X$-cs-model and $\mathcal{M}$ is a bounded morphic image of the submodel of $t_{3}(\mathcal{M})$ generated by $W_{3}^{\prime}=\{((x, y), n): x P y, n<\omega\}$ :

- the existence of an initial family and the admissibility of $V_{3}$ are dealt as before (in this case the generator is $\{(x, n): n<\omega\}$ ).

Let us check that it satisfies coherence. Given $(x, i),(y, j),(z, k) \in W_{3}$. Let us assume that we have $(x, i) R_{3}(y, j),(x, i) R_{3}(z, k)$ and $(y, j) P_{3}(z, k)$ theny $=$ $\left(y_{1}, w\right), z=\left(z_{1}, w\right)$ and $j=k$. So, or $i=j=k$, in which case, $x \in W$ and $y_{1}=x=z_{1}$; or $i<j=k$ and $y_{1}=w=z_{1}$. 
- let $f: W_{3}^{\prime} \rightarrow W$ be defined by: $\left.f((x, y), i)\right)=x$.

It is straightforward to see that it is a function let us see that it is also a p-morphism (the condition in $V$ is dealt as before):

- $f$ is a p-morphism in $R$

* $\left(\left(x_{1}, x_{2}\right), i\right) R_{3}\left(\left(y_{1}, y_{2}\right), j\right)$ then

$$
f\left(\left(\left(x_{1}, x_{2}\right), i\right)\right)=x_{1} R y_{1}=f\left(\left(\left(y_{1}, y_{2}\right), j\right)\right),
$$

* $f\left(\left(\left(x_{1}, x_{2}\right), i\right)\right) R y$ then $\left(\left(x_{1}, x_{2}\right), i\right) R_{3}((y, y), j)$ for any $j>i$;

- $f$ is a p-morphism in $P$

* $\left(\left(x_{1}, x_{2}\right), i\right) P_{3}\left(\left(y_{1}, y_{2}\right), j\right)$ then

$$
f\left(\left(\left(x_{1}, x_{2}\right), i\right)\right)=x_{1} P x_{2}=y_{2} P y_{1}=f\left(\left(\left(y_{1}, y_{2}\right), j\right)\right),
$$

* $f\left(\left(x_{1}, x_{2}\right), i\right) P y$ then $\left(\left(x_{1}, x_{2}\right), i\right) P_{3}\left(\left(y, x_{2}\right), i\right)$.

4. Let us consider the morphism as in 3., we just need to check the conditions involving $R_{4}$ :

- initiality is dealt as in 3 .

Coherence: Let us assume that we have $(x, i) R_{4}(y, j),(x, i) R_{4}(z, k)$ and $(y, j) P_{4}(z, k)$. Then $y=\left(y_{1}, w\right), z=\left(z_{1}, w\right)$ and $j=k$. If $x \in W$ then, if $i=j=k$ then $x=y_{1}=z_{1}$ and if $i<j=k$ then $y_{1}=w=z_{1}$. Otherwise $x=\left(x_{1}, x_{2}\right)$ and $y_{1}=t\left(x_{1}, w\right)=z_{1}$.

- $\left(\left(x_{1}, x_{2}\right), i\right) R_{4}\left(\left(y_{1}, y_{2}\right), j\right)$ then $y_{1}=t^{\prime}\left(x_{1}, y_{2}\right)$ hence

$$
f\left(\left(\left(x_{1}, x_{2}\right), i\right)\right)=x_{1} R y_{1}=f\left(\left(\left(y_{1}, y_{2}\right), j\right)\right),
$$

- $f\left(\left(\left(x_{1}, x_{2}\right), i\right)\right) R y$ then $t^{\prime}\left(x_{1}, y\right)=y$ thus $\left(\left(x_{1}, x_{2}\right), i\right) R_{4}((y, y), j)$ for any $j>i$.

We start by analysing the subclasses of reactive frames (or models) that correspond to the usual axioms for reflexivity and transitivity (with one operator).

Let us prove a lemma that will be useful throughout the paper:

Lemma 3.7 Given a cs-frame $(W, R, P)$ admitting as initial family I. Let $\gamma$ s and $\gamma^{\prime} s$ be two I-initial paths,

$$
l_{I}(\gamma s) \sim_{\left(I, \Delta_{I}\right)} l_{I}\left(\gamma^{\prime} s\right) .
$$

Proof. $l_{I}(\gamma s) \alpha \in \Delta_{I}$ iff there is a $\beta$ s.t. $\gamma s \beta$ is an I-initial path (iff $\gamma^{\prime} s \beta$ is an I-initial path) and $l_{I}(\beta)=\alpha$ iff $l_{I}\left(\gamma^{\prime} s\right) \alpha \in \Delta_{I}$. Since clearly $t\left(l_{I}(\gamma s)\right)=l_{I}(s)=t\left(l_{I}\left(\gamma^{\prime} s\right)\right)$ we get $l_{I}(\gamma s) \sim l_{I}\left(\gamma^{\prime} s\right)$.

Proposition 3.8 1. A reactive frame $(W, \Delta)(\Pi)$-validates $p \rightarrow \diamond_{R} p$ iff it is strongly reflexive, i.e. satisfies:

$$
\lambda w \in \Delta \rightarrow \lambda w \sim \lambda w w \in \Delta
$$

Let $L_{X}^{T}=L_{X} \oplus p \rightarrow \diamond_{R} p$. 
2. $L_{\Pi}^{T}$ is sound and complete with respect to the class of (all П-reactive models over) strongly reflexive frames.

3. If $X \neq \Pi, L_{X}^{T}$ is not sound and complete with respect to (all $X$-models over) any class of reactive frames.

Proof.

1.

- Given a strongly reflexive $\Pi$-reactive model $\mathcal{M}=(W, \Delta, v)$ and $\lambda w \in \Delta$ s.t. $\mathcal{M}, \lambda w \vDash_{\Pi} \varphi$. Thus $\lambda w \sim \lambda w w$ and, by proposition $2.5, \mathcal{M}, \lambda w w \vDash_{\Pi} \varphi$ and so $\mathcal{M}, \lambda w \models_{\Pi} \diamond_{R} \varphi$.

- Given a non strongly reactive frame $(W, \Delta)$. There exists some $\lambda w \in \Delta$ s.t. $\lambda w \nsim$ $\lambda w w$, so we have two cases.

Or $\lambda w w \notin \Delta$ so if we take $p \in \Pi$ and pick $v$ s.t. $\lambda w \in v(p)$ and $\lambda w w^{\prime} \notin v(p)$ for all $\lambda w w^{\prime} \in \Delta$ in which case

$$
(W, \Delta, v), \lambda w \vDash p \wedge \neg \diamond_{R} p .
$$

Or else, exists $\beta=w_{1} \ldots w_{n}$ s.t. we don't have $\lambda w \beta \in \Delta \leftrightarrow \lambda w w \beta \in \Delta$. We consider $w_{0}=t(\lambda)=t(\gamma), p_{0}, \ldots, p_{n}$ and pick $v$ s.t. $\alpha \in v\left(p_{i}\right)$ iff $t(\alpha)=w_{i}$. Let $\varphi=\diamond_{R} \varphi_{\beta}$ be as defined in proposition (2.5.2). If $\lambda w \beta \in \Delta$ but $\lambda w w \beta \notin \Delta$ then $\lambda w$ satisfies $\psi=\left(p_{0} \wedge \varphi\right) \vee \neg p_{0}$ and $\lambda w w$ does not. If $\lambda w \beta \notin \Delta$ but $\lambda w w \beta \in \Delta$ then $\lambda w$ satisfies $\neg \psi=\neg\left(p_{0} \wedge \varphi\right) \wedge p_{0}$ and $\lambda w w$ does not. Since no $\lambda w w^{\prime} \in \Delta$ with $w^{\prime} \neq w$ satisfies $p_{0}$ and both $\psi$ and $\neg \psi$ imply $p_{0}$ (which is only satisfied at paths with $w$ as end point), we know that either $(W, \Delta, v), \lambda w \forall \psi \rightarrow \diamond_{R} \psi$ or $(W, \Delta, v), \lambda w \not \models \neg \psi \rightarrow \diamond_{R} \neg \psi$

Imposing the usual axiom for reflexivity forces a very strong notion of reflexivity in reactive frames. Strong reflexivity imposes that, no matter which path we have covered, we can always loop without any change to the accessible worlds.

2. Soundness of $L_{\Pi}^{T}$ comes easily from the soundness of the new axiom which has just been established.

Let us prove that $L_{\Pi}^{T}=\{\varphi: \varphi$ is valid in every strongly reflexive reactive frame $\}$ :

- using lemma (3.2) it is easy to check that the canonical model for $L_{\Pi}^{T}$ is a $\Pi$ shattered model $(W, R, P, V)$ where $R$ reflexive. Since every $c s$-frame is also a shattered frame we have that:

$$
\begin{aligned}
L_{\Pi}^{T} & =\{\varphi: \varphi \text { is true in every } \Pi \text {-shattered model }(W, R, P, v) \text { where } R \text { reflexive }\} \\
& \subseteq\{\varphi: \varphi \text { is true in every } \Pi \text {-cs model }(W, R, P, v) \text { where } R \text { reflexive }\}
\end{aligned}
$$

- $t_{1}$ preserves $R$-reflexivity:

Given $(x, i)$ the case $i=*$ is trivial and if $i=\left(x^{\prime}, a\right)$ we have

$$
\left(x,\left(x^{\prime}, a\right)\right) R_{2}\left(t^{\prime}(x, x),\left(x^{\prime}, a\right)\right)=\left(x,\left(x^{\prime}, a\right)\right)
$$

since $t^{\prime}(x, x)=x$. Hence

$$
L_{\Pi}^{T}=\{\varphi: \varphi \text { is true in every } \Pi \text {-cs model }(W, R, P, v) \text { where } R \text { reflexive }\}
$$


- given a $R$-reflexive $c s$-frame $F=(W, R, P)$ with initial family $I,\left(I, \Delta^{I}\right)$ is a strongly reflexive reactive frame:

Let $\lambda w \in \Delta^{I}$, so there is $\gamma \in W^{*}$ and $s \in W$ s.t. $\gamma s$ is an I-initial path and $l_{I}(\gamma s)=\lambda w$. Since $F$ is $R$-reflexive we have that $\gamma s s$ is also an I-initial path and clearly $l_{I}(\gamma s s)=\lambda w w \in \Delta$. Applying lemma 3.7 we conclude that $\lambda w \sim \lambda w w$.

3. Let $X \subsetneq \Pi$ and a class of reactive frames $\mathcal{F}$.

If there is a reactive frame $F=(W, \Delta) \in \mathcal{F}$ then let $\lambda \in \Delta$ and $p \in \Pi-X$. We pick $v$ s.t. $\lambda \in v(p)$ and $\lambda w \notin v(p)$ for all $\lambda w \in \Delta$. So

$$
(W, \Delta, v), \lambda w \vDash p \wedge \neg \diamond_{R} p .
$$

If $\mathcal{F}$ is the empty class it validates $\perp$. Since there are $\Pi$-reactive models over strongly reflexive frames and $L_{X}^{T} \subseteq L_{\Pi}^{T}$ we conclude that

$$
L_{X}^{T} \subsetneq\{\varphi: \varphi \text { is valid in all } F \in \mathcal{F}\} \ni \perp \text {. }
$$

Notice that to have reactive frame completeness we have to impose that $X=\Pi$. This will also happen in the next case.

Proposition 3.9 1. A reactive frame $(W, \Delta)$ (П-)validates $\diamond_{R} \diamond_{R} p \rightarrow \diamond_{R} p$ iff it is strongly transitive, i.e. satisfies:

$$
\lambda w w^{\prime} w^{\prime \prime} \in \Delta \rightarrow \lambda w w^{\prime} w^{\prime \prime} \sim \lambda w w^{\prime \prime} \in \Delta
$$

Let $L_{X}^{4}=L_{X} \oplus \diamond_{R} \diamond_{R} p \rightarrow \diamond_{R} p$.

2. $L_{\Pi}^{4}$ is sound and complete with respect to the class of $\Pi$-reactive models over strongly transitive frames.

3. If $X \neq \Pi, L_{X}^{4}$ is not sound and complete with respect to (all $X$-models over) any class of reactive frames.

Proof. 1.

- Given a strongly transitive reactive model $\mathcal{M}=(W, \Delta, v)$ and $\lambda w \in \Delta$ s.t. $\mathcal{M}, \lambda w \models$ $\diamond_{R} \diamond_{R} \varphi$ then there are $w^{\prime}, w^{\prime \prime} \in W$ s.t. $\lambda w w^{\prime} w^{\prime \prime} \in \Delta$ and $\mathcal{M}, \lambda w w^{\prime} w^{\prime \prime} \vDash \varphi$. Thus $\lambda w w^{\prime} w^{\prime \prime} \sim \lambda w w^{\prime \prime} \in \Delta$ and, by proposition $2.5, \mathcal{M}, \lambda w w^{\prime \prime} \vDash \varphi$ hence $\mathcal{M}, \lambda w \vDash \diamond_{R} \varphi$.

- Given a non strongly transitive reactive frame $(W, \Delta)$. There exists some $\lambda w w^{\prime} w^{\prime \prime}$ $\in \Delta$ s.t. $\lambda w w^{\prime} w^{\prime \prime}+\lambda w w^{\prime \prime}$ then we have two cases:

Or $\lambda w w^{\prime \prime} \notin \Delta$ which implies $w^{\prime \prime} \neq w^{\prime}$. Hence, if we take $p \in \Pi$ and pick $v$ s.t. $\lambda w w^{\prime} w^{\prime \prime} \in v(p)$ and $\lambda w v \notin v(p)$ for all $\lambda w v \in \Delta$, in which case

$$
\lambda w \vDash \diamond_{R} \diamond_{R} p \wedge \neg \diamond_{R} p .
$$

Or exists $\beta$ s.t. we don't have $\lambda w w^{\prime} w^{\prime \prime} \beta \in \Delta \leftrightarrow \lambda w w^{\prime \prime} \beta \in \Delta$. In which case we pick $w_{0}=w^{\prime \prime}$ and define $\psi$ as in proposition (3.8.1). As before we conclude that either $(W, \Delta, v), \lambda w \not \models \diamond_{R} \diamond_{R} \psi \rightarrow \diamond_{R} \psi$ or $(W, \Delta, v), \lambda w \not \models \diamond_{R} \diamond_{R} \neg \psi \rightarrow \diamond_{R} \neg \psi$. 
Similarly to the case of strong reflexivity, strong transitivity imposes that, regardless of the path we have covered, every world accessible in two steps is accessible in one and that the set of accessible worlds is the same in both cases.

2. Soundness of $L_{\Pi}^{4}$ comes easily from the soundness of the new axiom which has just been established.

Let us prove that $L_{\Pi}^{4}=\{\varphi: \varphi$ is valid in every strongly reflexive reactive frame $\}$ :

- using lemma (3.2) it is easy to check that the canonical model for $L_{X}^{4}$ is a shattered model $(W, R, P, V)$ where $R$ transitive.

- $t_{3}$ preserves $R$-transitivity:

If $\left(x_{1}, i_{1}\right) R_{3}\left(x_{2}, i_{2}\right) R_{3}\left(x_{3}, i_{3}\right)$ then $x_{k}=\left(x_{k, 1}, x_{k, 2}\right)$ for $k=2,3, i_{1} \leq i_{2}<i_{3}$ and $x_{3,1}=x_{3,2}$. If $x_{1} \in W$ then immediately we conclude $\left(x_{1}, i_{1}\right) R_{3}\left(x_{3}, i_{3}\right)$. If $x_{1}=$ $\left(x_{1,1}, x_{1,2}\right)$ then $x_{1,1} R x_{2,1} R x_{3,1}$. Hence $x_{1,1} R x_{3,1}=x_{3,2}$ and $\left(x_{1}, i_{1}\right) R_{3}\left(x_{3}, i_{3}\right)$.

- given a $R$-transitive $c s$-frame with initial family $I,\left(I, \Delta^{I}\right)$ is a strongly transitive reactive frame:

Let $\lambda w w^{\prime} w^{\prime \prime} \in \Delta^{I}$, so there is $\gamma \in W^{*}$ and $s, s^{\prime}, s^{\prime \prime} \in W$ s.t. $\gamma s s^{\prime} s^{\prime \prime}$ is an I-initial path and $l_{I}\left(\gamma s s^{\prime} s^{\prime \prime}\right)=\lambda w w^{\prime} w^{\prime \prime}$. Since $F$ is $R$-transitive we have that $\gamma s s^{\prime \prime}$ is also an I-initial path and clearly $l_{I}\left(\gamma s s^{\prime \prime}\right)=\lambda w w^{\prime \prime} \in \Delta$. Applying lemma 3.7 we conclude that $\lambda w w^{\prime} w^{\prime \prime} \sim \lambda w w^{\prime \prime}$.

3. Let $X \subsetneq \Pi$ and a class of reactive frames $\mathcal{F}$.

If there is a reactive frame $F=(W, \Delta) \in \mathcal{F}$ with a path of length three, $w_{0} w_{1} w_{2}$, let $v$ be s.t. $w_{0} w_{1} w_{2} \in v(p)$ and $w_{0} v \notin v(p)$ for all $w_{0} v \in \Delta$ then $(W, \Delta, v), w_{0} \vDash$ $\diamond_{R} \diamond_{R} p \wedge \neg \diamond_{R} p$.

Given a reactive frame $(W, \Delta)$ s.t. there are no $w_{0}, w_{1}, w_{2} \in W$ s.t. $w_{0} w_{1} w_{2} \in \Delta$ then it validates $\diamond_{R} \square_{R} \perp$ but $\diamond_{R} \square_{R} \perp \notin L_{X}^{T}$ since

$$
L_{X}^{4} \subseteq L_{\Pi}^{4} \subseteq\left\{\varphi:\left(\{a\},\{a\}^{*}, v\right) \models \varphi \& v(p)=\{a\}^{*} \text { for all } p \in \Pi\right\} \not \diamond_{R} \square_{R} \perp .
$$

So if there is no frame with a path of length three in $\mathcal{F}$ (in particular if $\mathcal{F}$ is empty) then

$$
L_{X}^{4} \subsetneq\{\varphi: \varphi \text { is valid in all } F \in \mathcal{F}\} .
$$

Let us consider some variants of these axioms and see that they axiomatise other generalized notions of reflexivity and transitivity.

Proposition 3.10 1. A reactive frame $(W, \Delta) X$-validates $p \rightarrow \diamond_{R} \diamond_{P} p$ iff it is outwardly reflexive, i.e. satisfies:

$$
\lambda w \in \Delta \rightarrow \lambda w w \in \Delta .
$$

$L_{X}^{T_{o}}=L_{X} \oplus p \rightarrow \diamond_{R} \diamond_{P} p$ is sound and complete with respect to the class of $X$-reactive models over outwardly reflexive frames. 
2. Let $L_{X}^{T_{i}}=L_{X} \oplus p \rightarrow \diamond_{P} \diamond_{R} p$. If $X=\Pi, L_{\Pi}^{T_{i}}$ is sound and complete with respect to the class of П-reactive models over inwardly reflexive frames, i.e. satisfying:

$$
\lambda w \in \Delta \rightarrow \exists \lambda^{\prime} \lambda w \sim \lambda^{\prime} w w \in \Delta .
$$

If $X \subsetneq \Pi, L_{X}^{T_{i}}$ is not sound with respect to (all X-models over) any class of reactive frames.

3. A reactive frame $(W, \Delta) X$-validates $\square_{P} p \rightarrow \diamond_{P} \diamond_{R} p$ iff is lightly reflexive, i.e. satisfies:

$$
w \in W \rightarrow \exists \lambda \lambda w w
$$

$L_{X}^{T_{l}}=L_{X} \oplus \square_{P} p \rightarrow \diamond_{P} \diamond_{R} p$ is sound and complete with respect to the class of $X$-reactive models over inwardly reflexive frames.

It is clear that strong reflexivity implies inward and outward reflexivity and both imply light reflexivity.

\section{Proof.}

1. Given a $X$-reactive model $\mathcal{M}=(W, \Delta, v)$ over a outwardly reflexive reactive frame and $\lambda w \in \Delta$ s.t. $\mathcal{M}, \lambda w \vDash \varphi$ then since $\lambda w w \in \Delta$ and $t(\lambda w)=t(\lambda w w)$ we have $\mathcal{M}, \lambda w w \vDash \diamond_{P} \varphi$ and so $\mathcal{M}, \lambda w \vDash \diamond_{R} \diamond_{P} \varphi$.

If a reactive frame $(W, \Delta)$ is not outwardly reflexive then there exists some $\lambda w \in \Delta$ s.t. $\lambda w w \notin \Delta$. Picking a $p \in \Pi$ and choosing a $v$ s.t. $\lambda^{\prime} \in v(p)$ iff $t\left(\lambda^{\prime}\right)=w$. Now we have that $(W, \Delta, v), \lambda w \models p \wedge \neg \diamond_{R} \diamond_{P} p$.

Soundness of $L_{X}^{T_{o}}$ comes easily from the soundness of the new axiom which has just been established.

Using the same strategy as before we will obtain the equality:

$$
L_{X}^{T_{o}}=\{\varphi: \varphi \text { is } X \text {-valid in every outwardly reflexive reactive frame }\} .
$$

- $q \rightarrow \diamond_{R} \diamond_{P} q$ is a Sahlqvist formula and so the canonical frame $(W, R, P)$ for $L_{X}^{T_{o}}$ satisfies:

$$
\forall w \exists w^{\prime} w R w^{\prime} P w
$$

since it is the first-order correspondent to the new axiom.

- $t_{1}$ preserves property (a):

Given $(x, i)$, if $i=*$ then $(x, i) R_{1}(x, i) P_{1}(x, i)$ and if $i=\left(x^{\prime}, a\right)$ then by property (a) there exists $y$ s.t. $x R y P x$ thus $t^{\prime}(x, x)$ is defined and $(x, i) R_{1}\left(t^{\prime}(x, x), i\right) P_{1}(x, i)$.

- let $(W, R, P)$ be a cs-frame satisfying property (a) and $I$ an initial family. $\left(I, \Delta^{I}\right)$ is a outward reflexive reactive frame:

Let $\lambda w \in \Delta^{I}$ then there are $\gamma \in W^{*}$ and $s \in W$ s.t. $\gamma s$ is an I-initial path and $l_{I}(\gamma s)=\lambda w$. By property (a) there exists $t \in W$ s.t. $s R t P s$ (which implies $t P w$ ) thus $\gamma$ st is also an I-initial path and $l_{I}(\gamma s t)=\lambda w w \in \Delta^{I}$. 
2. To establish soundness we have just to check if the added axiom is sound: given a $\Pi$-reactive model $\mathcal{M}=(W, \Delta, v)$ over a inwardly reflexive reactive frame and $\lambda w \in \Delta$ s.t. $\mathcal{M}, \lambda w \vDash \varphi$ then since exists $\lambda^{\prime}$ s.t. $\lambda^{\prime} w w \in \Delta$ and $\lambda w \sim \lambda^{\prime} w w$. Thus by proposition (2.5) we have $\mathcal{M}, \lambda^{\prime} w w \vDash \varphi$ and since $t(\lambda w)=t\left(\lambda^{\prime} w\right)$ we have that $\mathcal{M}, \lambda w \vDash \diamond_{P} \diamond_{R} \varphi$.

For completeness we proceed as before:

- $p \rightarrow \diamond_{P} \diamond_{R} q$ is a Sahlqvist formula and so the canonical frame $F=(W, R, P)$ for $L_{\Pi}^{T_{i}}$ satisfies:

$$
\forall w \exists w^{\prime} w P w^{\prime} R w
$$

since it is its first-order correspondent.

- $t_{2}$ preserves property (b): given $w=(a, b, c, d, i) \in W_{2}$, if $i=*$ then $w P_{2} w R_{2} w$ and if $i \neq *$ then $(a, b, c, d, i) P_{2}(t(a), b+1, c, d, i) R_{2}(a, b, c, d, i)$.

- let $(W, R, P)$ be a cs-frame satisfying property (b) and I an initial family. $\left(I, \Delta^{I}\right)$ is a inwardly reflexive reactive frame:

Let $\lambda w \in \Delta^{I}$, so there is $\gamma \in W^{*}$ and $s \in W$ s.t. $\gamma s$ is an I-initial path and $l_{I}(\gamma s)=\lambda w$. By property (b) there exists $t \in W$ s.t. $t R s P t$. From the initiality of $I$ we know that there is some $\gamma^{\prime}$ s.t. $\gamma^{\prime} t$ is an I-initial path, thus $\gamma^{\prime} t s$ is also an $I$-initial and $l_{I}(\gamma s t)=l_{I}(\gamma) w w=\lambda^{\prime} w w \in \Delta^{I}$. Applying lemma 3.7 we conclude that $\lambda w \sim \lambda^{\prime} w w$.

So

$$
L_{\Pi}^{T_{i}}=\{\varphi: \varphi \text { is valid in every inwardly reflexive reactive frame }\} .
$$

Given $X \subsetneq \Pi$ and a reactive frame $(W, \Delta)$ there is a $X$-reactive model $\mathcal{M}=(W, \Delta, v)$, $\lambda \in \Delta$ and $\varphi$ s.t. $\mathcal{M}, \lambda \not \varphi \rightarrow \diamond_{R} \varphi$ : given $\lambda w \in \Delta$ and some $p \in \Pi-X$, let $v$ s.t. $\lambda w \in v(p)$ and $\lambda^{\prime} w w \notin v(p)$ for all $\lambda^{\prime} w w \in \Delta$. So

$$
(W, \Delta, v), \lambda w \vDash p \wedge \neg \diamond_{R} \diamond p
$$

3. Let $\mathcal{M}=(W, \Delta, v)$ be a $X$-reactive model over a lightly reflexive reactive frame and $\lambda w \in \Delta$ s.t. $\mathcal{M}, \lambda w \vDash \square_{P} \varphi$. Since there exists $\lambda^{\prime}$ s.t. $\lambda^{\prime} w w \in \Delta$ and $t(\lambda w)=t\left(\lambda^{\prime} w w\right)$ we have $\mathcal{M}, \lambda^{\prime} w w \vDash \varphi$ and so $\mathcal{M}, \lambda^{\prime} w \vDash \diamond_{R} \varphi$ hence $\mathcal{M}, \lambda w \vDash \diamond_{P} \diamond_{R} \varphi$.

If a reactive frame $(W, \Delta)$ is not lightly reflexive then there exists some $w \in W$ for which there is no $\lambda \in \Delta$ s.t. $\lambda w w \in \Delta$. So we pick a $p \in \Pi$ and choose a $v$ s.t. $\lambda^{\prime} \in v(p)$ iff $t\left(\lambda^{\prime}\right)=w$. Now we have that $(W, \Delta, v), w \models \square_{P} p \wedge \neg \diamond_{P} \diamond_{R} p$.

Soundness of $L_{X}^{T_{i}}$ comes easily from the soundness of the new axiom which has just been established.

For completeness, as before:

- $\square p \rightarrow \diamond_{P} \diamond_{R} q$ is a Sahlqvist formula and (just as before) the canonical model $(W, R, P)$ for $L_{X}^{T_{o}}$ satisfies:

$$
\forall w \exists w^{\prime} w^{\prime \prime} w P w^{\prime} R w^{\prime \prime} P w
$$

since it is its first-order correspondent. 
- $t_{1}$ preserves property (c): given $(x, i) \in W_{1}$, if $i=1$ then

$$
(x, i) P_{1}(x, i) R_{1}(x, i) P_{1}(x, i)
$$

and if $i=\left(x^{\prime}, a\right)$ then by property (c) there exist $y, z$ s.t. $x P y R z P x$ thus $t^{\prime}(y, y)$ is defined and

$$
(x, i) P_{1}(y, i) R_{1}(t(y, y), i) P_{1}(x, i) .
$$

- let $(W, R, P)$ be a cs-frame satisfying property (c) and I an initial family. $\left(I, \Delta^{I}\right)$ is a lightly reflexive reactive frame:

Let $\lambda w \in \Delta^{I}$ then there are $s, t$ s.t. $l_{I}(s)=w=l_{I}(t)$ and $s R t$. By initiality of $I$ there is an I-initial path ending in $s, \gamma s$, making $\gamma$ st also an I-initial path, hence $l_{I}(\gamma) w w=\lambda^{\prime} w w \in \Delta$.

So

$$
L_{X}^{T_{l}}=\{\varphi: \varphi \text { is } X \text {-valid in every lightly reflexive reactive frame }\}
$$

Proposition 3.11 1. A reactive frame $(W, \Delta) X$-validates $\diamond_{R} \diamond_{R} p \rightarrow \diamond_{R} \diamond_{P} p$ iff it is left transitive, i.e. satisfies:

$$
\lambda w w^{\prime} w^{\prime \prime} \in \Delta \rightarrow \lambda w w^{\prime \prime} \in \Delta
$$

$L_{X}^{4_{l}}=L_{X} \oplus \diamond_{R} \diamond_{R} p \rightarrow \diamond_{R} \diamond_{P} p$ is sound and complete with respect to the class of $X$-reactive models over left transitive reactive frames.

2. A reactive frame $(W, \Delta) X$-validates $\diamond_{R} \diamond_{R} p \rightarrow \diamond_{P} \diamond_{R} \diamond_{P} p$ iff it is middle transitive, i.e. satisfies:

$$
\lambda w w^{\prime} w^{\prime \prime} \in \Delta \rightarrow \exists \lambda^{\prime} \lambda^{\prime} w w^{\prime \prime} \in \Delta
$$

$L_{X}^{4_{m}}=L_{X} \oplus \diamond_{R} \diamond_{R} p \rightarrow \diamond_{P} \diamond_{R} \diamond_{P} p$ is sound and complete with respect to the class of $X$-reactive models over middle transitive reactive frames.

3. Let $L_{X}^{4_{r}}=L_{X} \oplus \diamond_{R} \diamond_{R} p \rightarrow \diamond_{P} \diamond_{R} p$. $L_{\Pi}^{4_{r}}$ is sound and complete with respect to the class of П-reactive models over right transitive reactive frames, i.e. satisfying:

$$
\lambda w w^{\prime} w^{\prime \prime} \in \Delta \rightarrow \exists \lambda^{\prime} \lambda w w^{\prime} w^{\prime \prime} \sim \lambda^{\prime} w w^{\prime \prime} \in \Delta
$$

If $X \subsetneq \Pi, L_{X}^{4_{r}}$ is not sound and complete with respect to (all $X$-models over) any class of reactive frames.

4. A reactive frame $(W, \Delta) X$-validates $\diamond_{R} \diamond_{P} \diamond_{R} p \rightarrow \diamond_{R} \diamond_{P} p$ iff it is globally left transitive, i.e. satisfies:

$$
\lambda w w^{\prime}, \lambda^{\prime} w^{\prime} w^{\prime \prime} \in \Delta \rightarrow \lambda w w^{\prime \prime} \in \Delta
$$

$L_{X}^{4_{g l}}=L_{X} \oplus \diamond_{R} \diamond_{P} \diamond_{R} p \rightarrow \diamond_{R} \diamond_{P} p$ is sound and complete with respect to the class of $X$-reactive models over globally left transitive reactive frames. 
5. A reactive frame $(W, \Delta) X$-validates $\diamond_{R} \diamond_{P} \diamond_{R} p \rightarrow \diamond_{P} \diamond_{R} \diamond_{P} p$ iff it is globally middle transitive, i.e. satisfies:

$$
\lambda w w^{\prime}, \lambda^{\prime} w^{\prime} w^{\prime \prime} \in \Delta \rightarrow \exists \lambda^{\prime \prime} \lambda^{\prime \prime} w w^{\prime \prime} \in \Delta .
$$

$L_{X}^{4_{g m}}=L_{X} \oplus \diamond_{R} \diamond_{P} \diamond_{R} p \rightarrow \diamond_{P} \diamond_{R} \diamond_{P} p$ is sound and complete with respect to the class of $X$-reactive models over globally transitive reactive frames.

6. Let $L_{X}^{4_{g r}}=L_{X} \oplus \diamond_{R} \diamond_{P} \diamond_{R} p \rightarrow \diamond_{P} \diamond_{R} p$. $L_{\Pi}^{4_{g r}}$ is sound and complete with respect to the class of $\Pi$-reactive models over globally right transitive reactive frames, i.e. satisfies:

$$
\lambda w w^{\prime}, \lambda^{\prime} w^{\prime} w^{\prime \prime} \in \Delta \rightarrow \exists \lambda^{\prime \prime} \lambda^{\prime} w^{\prime} w^{\prime \prime} \sim \lambda^{\prime \prime} w w^{\prime \prime} \in \Delta
$$

If $X \subsetneq \Pi, L_{X}^{4_{g r}}$ is not sound and complete with respect to (all $X$-models over) any class of reactive frames.

It is clear that strong transitivity implies left, middle and right transitivity. Both left and right transitivity imply middle transitivity and all of them are implied by its global version.

\section{Proof.}

1. Given a $X$-reactive model $\mathcal{M}=(W, \Delta, v)$ over a left transitive reactive frame and $\lambda w \in \Delta$ s.t.

$$
\mathcal{M}, \lambda w \vDash \diamond_{R} \diamond_{R} \varphi,
$$

i.e. there are $w^{\prime}, w^{\prime \prime}$ s.t. $\lambda w w^{\prime} w^{\prime \prime} \in \Delta$ and $\mathcal{M}, \lambda w w^{\prime} w^{\prime \prime} \vDash \varphi$. By left transitivity we get that $\lambda w w^{\prime \prime} \in \Delta$ and since $t\left(\lambda w w^{\prime} w^{\prime \prime}\right)=t\left(\lambda w w^{\prime \prime}\right)$ we have

$$
\mathcal{M}, \lambda w \vDash \diamond_{R} \diamond_{P} \varphi .
$$

If a reactive frame $(W, \Delta)$ is not left transitive then there exists $\lambda w w^{\prime} w^{\prime \prime} \in \Delta$ s.t. $\lambda w w^{\prime \prime} \notin$ $\Delta$. So we pick a $p \in \Pi$ and choose a $v$ s.t. $\gamma \in v(p)$ iff $t(\gamma)=w^{\prime \prime}$. Now we have that

$$
(W, \Delta, v), \lambda w \vDash \diamond_{R} \diamond_{R} p \wedge \neg \diamond_{R} \diamond_{P} p .
$$

Soundness of $L_{X}^{4_{l}}$ follows from the soundness of the new axiom which has just been established.

Let us prove that $L_{X}^{4_{l}}=\{\varphi: \varphi$ is $X$-valid in every left transitive reactive frame $\}$ :

- $\diamond_{R} \diamond_{R} p \rightarrow \diamond_{R} \diamond_{P} p$ is a Sahlqvist formula and so the canonical model $(W, R, P)$ for $L_{X}^{4_{l}}$ satisfies:

$$
\forall t v w \exists w^{\prime} t R v R w \rightarrow t R w^{\prime} P w
$$

since it is its first-order correspondent.

- $t_{4}$ preserves property $(L T)$ :

Given $\left(x_{k}, i_{k}\right) \in W_{4}$ for $k=1,2,3$ s.t. $\left(x_{1}, i_{1}\right) R_{4}\left(x_{2}, i_{2}\right) R_{4}\left(x_{3}, i_{3}\right)$. So $i_{1} \leq i_{2}<i_{3}$, $x_{k}=\left(x_{k, 1}, x_{k, 2}\right)$ for $k=2,3$. If $x_{1} \in W$ we have that

$$
\left(x_{1}, i_{1}\right) R_{4}\left(\left(x_{3,2}, x_{3,2}\right), i_{3}\right) P_{4}\left(x_{3}, i_{3}\right) .
$$


If $x_{1}=\left(x_{1,1}, x_{1,2}\right)$ then $x_{1,1} R x_{2,1} R x_{3,1}$ hence, by property $(L T)$, there is $x$ s.t. $x_{1,1} R x P x_{3,1}\left(P x_{3,2}\right)$. Thus $t^{\prime}\left(x_{1,1}, x_{3,2}\right)$ is defined and

$$
\left(x_{1}, i_{1}\right) R_{4}\left(\left(t^{\prime}\left(x_{1,1}, x_{3,2}\right)\right), i_{3}\right) P_{4}\left(x_{3}, i_{3}\right) .
$$

- let $(W, R, P)$ be a cs-frame satisfying property $(L T)$ and $I$ an initial family. $\left(I, \Delta^{I}\right)$ is a left transitive reactive frame:

Let $\lambda w w^{\prime} w^{\prime \prime} \in I$ then there are some $\gamma \in W^{*}$ and $s, s^{\prime}, s^{\prime \prime} \in W$ s.t. $\gamma s s^{\prime} s^{\prime \prime}$ is an $I$-initial path and $l_{I}\left(\gamma s s^{\prime} s^{\prime \prime}\right)=\lambda w w^{\prime} w^{\prime \prime}$. By $(L T)$ there exists some $t \in W$ s.t. $s R t P s^{\prime \prime} P w$ and so $\gamma$ st is an I-initial path. Hence $l_{I}(\gamma s t)=\lambda w w^{\prime \prime} \in \Delta^{I}$.

2. Given a $X$-reactive model $\mathcal{M}=(W, \Delta, v)$ over a middle transitive reactive frame and $\lambda w \in \Delta$ s.t.

$$
\mathcal{M}, \lambda w \models \diamond_{R} \diamond_{R} \varphi,
$$

i.e. there are $w^{\prime}, w^{\prime \prime}$ s.t. $\lambda w w^{\prime} w^{\prime \prime} \in \Delta$ and $\mathcal{M}, \lambda w w^{\prime} w^{\prime \prime} \vDash \varphi$. By middle transitivity, there is $\lambda^{\prime}$ s.t. $\lambda^{\prime} w w^{\prime \prime} \in \Delta$. Since $t\left(\lambda w w^{\prime} w^{\prime \prime}\right)=t\left(\lambda^{\prime} w w^{\prime \prime}\right)$ and $t(\lambda w)=t\left(\lambda^{\prime} w\right)$ we have

$$
\mathcal{M}, \lambda w \models \diamond_{P} \diamond_{R} \diamond_{P} \varphi
$$

If a reactive frame $(W, \Delta)$ is not middle transitive then there exists some $\lambda w w^{\prime} w^{\prime \prime} \in \Delta$ and no $\lambda^{\prime}$ s.t. $\lambda^{\prime} w w^{\prime \prime} \in \Delta$. So we pick a $p \in \Pi$ and choose a $v$ s.t. $\gamma \in v(p)$ iff $t(\gamma)=w^{\prime \prime}$. Obtaining

$$
(W, \Delta, v), \lambda w \vDash \diamond_{R} \diamond_{R} p \wedge \neg \diamond_{P} \diamond_{R} \diamond_{P} p .
$$

Soundness of $L_{X}^{4_{l}}$ follows from the soundness of the new axiom which has just been established.

We prove completeness as before:

- $\diamond_{R} \diamond_{R} p \rightarrow \diamond_{P} \diamond_{R} \diamond_{P} p$ is a Sahlqvist formula and so the canonical model $(W, R, P)$ for $L_{X}^{4_{m}}$ satisfies:

$$
\forall t v w \exists t^{\prime} w^{\prime} t R v R w \rightarrow t P t^{\prime} R w^{\prime} P w
$$

since it is its first-order correspondent.

- $t_{4}$ preserves property property $(M T)$ :

Given $\left(x_{k}, i_{k}\right) \in W_{4}$ for $k=1,2,3$ s.t. $\left(x_{1}, i_{1}\right) R_{4}\left(x_{2}, i_{2}\right) R_{4}\left(x_{3}, i_{3}\right)$. So $i_{1} \leq i_{2}<i_{3}$, $x_{k}=\left(x_{k, 1}, x_{k, 2}\right)$ for $k=2,3$. If $x_{1} \in W$ we have that

$$
\left(x_{1}, i_{1}\right) P_{4}\left(x_{1}, i_{1}\right) R_{4}\left(\left(x_{3,2}, x_{3,2}\right), i_{3}\right) P_{4}\left(x_{3}, i_{3}\right) .
$$

If $x_{1}=\left(x_{1,1}, x_{1,2}\right)$ then $x_{1,1} R x_{2,1} R x_{3,1}$ hence, by property $(M T)$, there are $x$, $x^{\prime}$ s.t. $x_{1,1} P x R x^{\prime} P x_{3,1}\left(P x_{3,2}\right)$ and so $t^{\prime}\left(x, x_{3,2}\right)$ is defined and

$$
\left(x_{1}, i_{1}\right) P_{4}\left(\left(x, x_{1,2}\right), i_{1}\right) R_{4}\left(\left(t^{\prime}\left(x, x_{3,2}\right)\right), i_{3}\right) P_{4}\left(x_{3}, i_{3}\right) .
$$

- let $(W, R, P)$ be a cs-frame satisfying property $(M T)$ and $I$ an initial family. $\left(I, \Delta^{I}\right)$ is a middle transitive reactive frame:

Let $\lambda w w^{\prime} w^{\prime \prime} \in I$ then there are some $\gamma \in W^{*}$ and $s, s^{\prime}, s^{\prime \prime} \in W$ s.t. $\gamma s s^{\prime} s^{\prime \prime}$ is an initial path and $l_{I}\left(\gamma s s^{\prime} s^{\prime \prime}\right)=\lambda w w^{\prime} w^{\prime \prime}$. From property (MT) follows that there are some $t, t^{\prime \prime} \in W$ s.t. $s P t R t^{\prime \prime} P s^{\prime \prime}$. By initiality of I there is an I-initial path $\gamma^{\prime} t$, making $\gamma^{\prime} t t^{\prime \prime}$ also an I-initial thus $l_{I}\left(\gamma^{\prime} t t^{\prime \prime}\right)=l_{I}\left(\gamma^{\prime}\right) w w^{\prime \prime}=\lambda^{\prime} w w^{\prime \prime} \in \Delta^{I}$. 
So $L_{X}^{4_{m}}=\{\varphi: \varphi$ is $X$-valid in every middle transitive reactive frame $\}$.

3. To estabilish soundness of $L_{\Pi}^{4_{r}}$ it is enough to verify soundness of the added axiom: given a $\Pi$-reactive model $\mathcal{M}=(W, \Delta, v)$ over a right transitive reactive frame and $\lambda w \in \Delta$ s.t.

$$
\mathcal{M}, \lambda w \vDash \diamond_{R} \diamond_{R} \varphi,
$$

so there are $w^{\prime}, w^{\prime \prime}$ s.t. $\lambda w w^{\prime} w^{\prime \prime} \in \Delta$ and $\mathcal{M}, \lambda w w^{\prime} w^{\prime \prime} \vDash \varphi$. By right transitivity there is $\lambda^{\prime} w w^{\prime \prime} \in \Delta$ s.t. $\lambda w w^{\prime} w^{\prime \prime} \sim \lambda^{\prime} w w^{\prime \prime}$ and so, by proposition $2.5, \mathcal{M}, \lambda^{\prime} w w^{\prime \prime} \vDash \varphi$. Thus

$$
\mathcal{M}, \lambda w \models \diamond_{P} \diamond_{R} \varphi .
$$

For completeness:

- $\diamond_{R} \diamond_{R} p \rightarrow \diamond_{P} \diamond_{R} p$ is a Sahlqvist formula and so the canonical model $(W, R, P)$ for $L_{\Pi}^{4_{r}}$ satisfies:

$$
\forall t v w \exists t^{\prime} t R v R w \rightarrow t P t^{\prime} R w
$$

since it is its first-order correspondent.

- $t_{3}$ preserves property $(R T)$ :

Given $\left(x_{k}, i_{k}\right) \in W_{3}$ for $k=1,2,3$ s.t. $\left(x_{1}, i_{1}\right) R_{3}\left(x_{2}, i_{2}\right) R_{3}\left(x_{3}, i_{3}\right)$. So $i_{1} \leq$ $i_{2}<i_{3}, x_{k}=\left(x_{k, 1}, x_{k, 2}\right)$ for $k=2,3$ and $x_{3,1}=x_{3,2}$. If $x_{1} \in W$ then immediately we conclude that $\left(x_{1}, i_{1}\right) P_{3}\left(x_{1}, i_{1}\right) R_{3}\left(x_{3}, i_{3}\right)$. If $x_{1}=\left(x_{1,1}, x_{1,2}\right)$ then $x_{1,1} R x_{2,1} R x_{3,1}=x_{3,2}$ so by property (RT) exists a $x$ s.t. $x_{1,1} P x R x_{3,1}$ and so $\left(x_{1}, i_{1}\right) P_{3}\left(\left(x, x_{1,2}\right), i_{1}\right) R_{3}\left(x_{3}, i_{3}\right)$.

- let $(W, R, P)$ be a cs-frame satisfying property $(R T)$ and $I$ an initial family. $\left(I, \Delta^{I}\right)$ is a right transitive reactive frame:

Let $\lambda w w^{\prime} w^{\prime \prime} \in \Delta^{I}$ then there is an I-initial path $\gamma s_{1} s_{2} s_{3}\left(s_{i} \in W\right)$ s.t. $l_{I}\left(\gamma s_{1} s_{2} s_{3}\right)=$ $\lambda w w^{\prime} w^{\prime \prime}$. By property (RT) exists $s \in W$ s.t. $s_{1} P s R s_{3}$. From the initiality of $I$ we know that there is some $\gamma^{\prime}$ s.t. $\gamma^{\prime} s$ is an I-initial path and so $\gamma^{\prime} s s_{3}$ is also an I-initial. Applying lemma 3.7 we conclude that $\Delta^{I} \ni l_{I}\left(\gamma^{\prime} s t\right)=l_{I}\left(\gamma^{\prime}\right) w w^{\prime \prime}=$ $\lambda^{\prime} w w^{\prime \prime} \sim \lambda w w^{\prime} w^{\prime \prime}$.

Hence,

$$
L_{\Pi}^{4_{r}}=\{\varphi: \varphi \text { is } \Pi \text {-valid in every right transitive reactive frame }\} .
$$

Given $X \subsetneq \Pi$ and a (non-empty) class of reactive frames $\mathcal{F}$ :

- if there is a reactive frame $(W, \Delta) \in \mathcal{F}$ with a path $\lambda w_{0} w_{1} w_{2}$, where $w_{0} \neq w_{1}$, we pick $p \in \Pi-X$ and $v$ s.t. $\gamma \in v(p)$ iff $\gamma=\lambda w_{0} w_{1} w_{2}$. Thus

$$
(W, \Delta, v), \lambda w_{0} \vDash \diamond_{R} \diamond_{R} p \wedge \neg \diamond_{P} \diamond_{R} p .
$$

- if $\mathcal{F}$ contains only reactive frames with paths of length bigger 2 of the form $\lambda w w w^{\prime}$ then it validates $\diamond_{R}\left(\varphi \wedge \diamond_{R} \top\right) \rightarrow \diamond_{P} \varphi$. Consider that $L_{X}^{4_{r}} \subseteq L_{\Pi}^{4_{r}}$ and the right transitive $\Pi$-reactive model: $\mathcal{M}=(\{0,1\},\{0,1,01,011\}, v)$ s.t. $\lambda \in v(p)$ iff $t(\lambda)=1$. It is clear that $\mathcal{M}, 0 \vDash \diamond_{R}\left(p \wedge \diamond_{R} T\right) \wedge \neg \diamond_{P} p$ and so:

$$
L_{X}^{4_{r}} \subsetneq\{\varphi: \varphi \text { is valid in all } F \in \mathcal{F}\} \text {. }
$$


- if $\mathcal{F}$ contains only reactive frames with paths of length smaller then 3 , following the same reasoning as in proposition (3.9.3), together with the fact that the strong transitive reactive frame used there - $\left(\{a\},\{a\}^{*}\right)$ - is also a right transitive reactive frame, we conclude that $L_{X}^{4_{r}}$ is not complete with respect to $\mathcal{F}$.

4. Given a $X$-reactive model $\mathcal{M}=(W, \Delta, v)$ over a globally left transitive reactive frame and $\lambda w \in \Delta$ s.t.

$$
\mathcal{M}, \lambda w \models \diamond_{R} \diamond_{P} \diamond_{R} \varphi,
$$

i.e. there are $w^{\prime}, w^{\prime \prime} \in W$ and $\lambda^{\prime}$ s.t. $\lambda w w^{\prime}, \lambda^{\prime} w^{\prime} w^{\prime \prime} \in \Delta$ and $\mathcal{M}, \lambda^{\prime} w^{\prime} w^{\prime \prime} \vDash \varphi$. By light left transitivity, there is $\lambda w w^{\prime \prime} \in \Delta$ and since $t\left(\lambda w w^{\prime \prime}\right)=t\left(\lambda^{\prime} w^{\prime} w^{\prime \prime}\right)$ we have

$$
\mathcal{M}, \lambda w \models \diamond_{R} \diamond_{P} \varphi
$$

If a reactive frame $(W, \Delta)$ is not globally left transitive then there exists some $\lambda w w^{\prime}$, $\lambda^{\prime} w^{\prime} w^{\prime \prime} \in \Delta$ s.t. $\lambda w w^{\prime \prime} \notin \Delta$. So we pick a $p \in \Pi$ and choose a $v$ s.t. $\gamma \in v(p)$ iff $t(\gamma)=w^{\prime \prime}$. Hence

$$
(W, \Delta, v), \lambda w \vDash \diamond_{R} \diamond_{P} \diamond_{R} p \wedge \neg \diamond_{R} \diamond_{P} p .
$$

Soundness of $L_{X}^{4_{l}}$ comes easily from the soundness of the new axiom which has just been established.

We establish completeness as before:

- $\diamond_{R} \diamond_{P} \diamond_{R} p \rightarrow \diamond_{R} \diamond_{P} p$ is a Sahlqvist formula and so the canonical model $(W, R, P)$ for $L_{X}^{4_{g l}}$ satisfies:

$$
\forall t v v^{\prime} w \exists w^{\prime} t R v P v^{\prime} R w \rightarrow t R w^{\prime} P w
$$

since it is its first-order correspondent.

- $t_{4}$ preserves property (LLT):

Given $\left(x_{k}, i_{k}\right) \in W_{4}$ for $k=1,2,3,4$ s.t. $\left(x_{1}, i_{1}\right) R_{4}\left(x_{2}, i_{2}\right) P_{4}\left(x_{3}, i_{3}\right) R_{4}\left(x_{4}, i_{4}\right)$. So $i_{1} \leq i_{2}=i_{3}<i_{4}, x_{k}=\left(x_{k, 1}, x_{k, 2}\right)$ for $k=2,3$, 4. If $x_{1} \in W$ we have that

$$
\left(x_{1}, i_{1}\right) R_{4}\left(\left(x_{4,2}, x_{4,2}\right), i_{4}\right) P_{4}\left(x_{4}, i_{4}\right) .
$$

If $x_{1}=\left(x_{1,1}, x_{1,2}\right)$ then $x_{1,1} R x_{2,1} P x_{3,1} R x_{4,1}$ hence, by property (LLT), there is $x$ s.t. $x_{1,1} R x P x_{4,1}\left(P x_{4,1}\right)$ and so $t^{\prime}\left(x_{1,1}, x_{4,2}\right)$ is defined and

$$
\left(x_{1}, i_{1}\right) R_{4}\left(\left(t^{\prime}\left(x_{1,1}, x_{4,2}\right)\right), i_{3}\right) P_{4}\left(x_{4}, i_{4}\right) .
$$

- given a cs-frame $(W, R, P)$ satisfying property $(L L T)$ with initial family $I,\left(I, \Delta^{I}\right)$ is a light left transitive reactive frame: Let $\lambda w w^{\prime}, \lambda^{\prime} w^{\prime} w^{\prime \prime} \in \Delta^{I}$ then there are $I$-initial paths $\gamma s_{1} s_{2}$ and $\gamma^{\prime} s_{3} s_{4}\left(s_{i} \in W\right)$ s.t. $l_{I}\left(\gamma s_{1} s_{2}\right)=\lambda w w^{\prime}$ and $l_{I}\left(\gamma^{\prime} s_{3} s_{4}\right)=$ $\lambda^{\prime} w^{\prime} w^{\prime \prime}$ (so $s_{1} R s_{2} P s_{3} R s_{4}$ ). By property (LLT) there exists $s \in W$ s.t. $s_{1} R s P s_{4}$. So $\gamma s_{1} s$ is also an I-initial path and $l_{I}\left(\gamma s_{1} s\right)=\lambda w w^{\prime \prime} \in \Delta^{I}$.

Hence

$$
L_{X}^{4_{g l}}=\{\varphi: \varphi \text { is } X \text {-valid in every light left transitive reactive frame }\} .
$$


5. Given a $X$-reactive model $\mathcal{M}=(W, \Delta, v)$ over a light transitive reactive frame and $\lambda w \in \Delta$ s.t.

$$
\mathcal{M}, \lambda w \models \diamond_{R} \diamond_{P} \diamond_{R} \varphi,
$$

i.e. there are $w^{\prime}, w^{\prime \prime} \in W$ and $\lambda^{\prime}$ s.t. $\lambda w w^{\prime}, \lambda^{\prime} w^{\prime} w^{\prime \prime} \in \Delta$ and $\mathcal{M}, \lambda^{\prime} w^{\prime} w^{\prime \prime} \vDash \varphi$. By globally middle transitivity, there is $\lambda^{\prime \prime}$ s.t. $\lambda^{\prime \prime} w w^{\prime \prime} \in \Delta$, thus

$$
\mathcal{M}, \lambda w \models \diamond_{P} \diamond_{R} \diamond_{P} \varphi
$$

If a reactive frame $(W, \Delta)$ is not globally middle transitive then there exists some $\lambda w w^{\prime}, \lambda^{\prime} w^{\prime} w^{\prime \prime} \in \Delta$ s.t. for all $\lambda^{\prime \prime}$ we have $\lambda^{\prime \prime} w w^{\prime \prime} \notin \Delta$. So we pick $p \in \Pi$ and $v$ s.t. $\gamma \in v(p)$ iff $t(\gamma)=w^{\prime \prime}$. Hence

$$
(W, \Delta, v), \lambda w \models \diamond_{R} \diamond_{P} \diamond_{R} p \wedge \neg \diamond_{P} \diamond_{R} \diamond_{P} p
$$

Soundness of $L_{X}^{4_{l}}$ comes easily from the soundness of the new axiom which has just been established.

Completeness:

- $\diamond_{R} \diamond_{P} \diamond_{R} p \rightarrow \diamond_{P} \diamond_{R} \diamond_{P} p$ is a Sahlqvist formula and so the canonical model $(W, R, P)$ for $L_{X}^{4_{l}}$ satisfies:

$$
\forall t, v, v^{\prime}, w \exists t^{\prime}, w^{\prime} t R v P v^{\prime} R w \rightarrow t P t^{\prime} R w^{\prime} P w
$$

since it is the first-order correspondent to the new axiom.

- $t_{4}$ preserves property $(G M)$ :

Given $\left(x_{k}, i_{k}\right) \in W_{4}$ for $k=1,2,3,4$ s.t. $\left(x_{1}, i_{1}\right) R_{4}\left(x_{2}, i_{2}\right) P_{4}\left(x_{3}, i_{3}\right) R_{4}\left(x_{4}, i_{4}\right)$. So $i_{1} \leq i_{2}=i_{3}<i_{4}, x_{k}=\left(x_{k, 1}, x_{k, 2}\right)$ for $k=2,3$, 4. If $x_{1} \in W$ we have that

$$
\left(x_{1}, i_{1}\right) P_{4}\left(x_{1}, i_{1}\right) R_{4}\left(\left(x_{4,2}, x_{4,2}\right), i_{4}\right) P_{4}\left(x_{4}, i_{4}\right) .
$$

If $x_{1}=\left(x_{1,1}, x_{1,2}\right)$ then $x_{1,1} R x_{2,1} P x_{3,1} R x_{4,1}$ hence, by property (GM), there is $x, x^{\prime}$ s.t. $x_{1,1} P x R x^{\prime} P x_{4,1}$ and so $t^{\prime}\left(x, x_{4,2}\right)$ is defined and

$$
\left(x_{1}, i_{1}\right) P_{4}\left(\left(x, x_{1,2}\right), i_{1}\right) R_{4}\left(\left(t^{\prime}\left(x, x_{4,2}\right)\right), i_{3}\right) P_{4}\left(x_{4}, i_{4}\right) .
$$

- given a cs-frame $(W, R, P)$ satisfying property $(G M)$ with initial family $I,\left(I, \Delta^{I}\right)$ is a globally middle transitive reactive frame:

Let $\lambda w w^{\prime}, \lambda^{\prime} w^{\prime} w^{\prime \prime} \in \Delta^{I}$ then there are I-initial paths $\gamma s_{1} s_{2}$ and $\gamma^{\prime} s_{3} s_{4}\left(s_{i} \in W\right)$ s.t. $l_{I}\left(\gamma s_{1} s_{2}\right)=\lambda w w^{\prime}$ and $l_{I}\left(\gamma^{\prime} s_{3} s_{4}\right)=\lambda^{\prime} w^{\prime} w^{\prime \prime}$ (in particular $\left.s_{1} R s_{2} P s_{3} R s_{4}\right)$. By property (GM) there exists $t, t^{\prime} \in W$ s.t. $s_{1} P t R t^{\prime} R s_{4}$. By initiality of $I$, there is some $\gamma^{\prime \prime}$ s.t. $\gamma^{\prime \prime} t$ is an I-initial path and so $\gamma^{\prime \prime} t t^{\prime}$ is also an I-initial path and $l_{I}\left(\gamma^{\prime \prime} t t^{\prime}\right)=\lambda^{\prime \prime} w w^{\prime \prime} \in \Delta^{I}$.

Hence $L_{X}^{4_{g m}}=\{\varphi: \varphi$ is $X$-valid in every globally middle transitive reactive frame $\}$.

6. To establish soundness of $L_{\Pi}^{4_{g r}}$ it is enough to verify soundness of the added axiom: given a $\Pi$-reactive model $\mathcal{M}=(W, \Delta, v)$ over a globally right transitive reactive frame and $\lambda w \in \Delta$ s.t.

$$
\mathcal{M}, \lambda w \vDash \diamond_{R} \diamond_{P} \diamond_{R} \varphi
$$


i.e. there are $w^{\prime}, w^{\prime \prime} \in W$ and $\lambda^{\prime}$ s.t. $\lambda w w^{\prime}, \lambda^{\prime} w^{\prime} w^{\prime \prime} \in \Delta$ and $\mathcal{M}, \lambda^{\prime} w^{\prime} w^{\prime \prime} \vDash \varphi$. By globally right transitivity we get that there is $\lambda^{\prime \prime}$ s.t. $\lambda^{\prime \prime} w w^{\prime \prime} \sim \lambda^{\prime} w^{\prime} w^{\prime \prime}$, so by proposition 2.5 we get $\mathcal{M}, \lambda^{\prime \prime} w w^{\prime \prime} \vDash \varphi$ and so

$$
\mathcal{M}, \lambda w \vDash \diamond_{P} \diamond_{R} \varphi
$$

For completeness:

- $\diamond_{R} \diamond_{P} \diamond_{R} p \rightarrow \diamond_{P} \diamond_{R} p$ is a Sahlqvist formula and so the canonical model $(W, R, P)$ for $L_{X}^{4_{g r}}$ satisfies:

$$
\forall t v v^{\prime} w \exists t^{\prime} t R v P v^{\prime} R w \rightarrow t P t^{\prime} R w
$$

since it is its first-order correspondent.

- $t_{3}$ preserves property $(G R)$ :

Given $\left(x_{k}, i_{k}\right) \in W_{3}$ for $k=1,2,3,4$ s.t. $\left(x_{1}, i_{1}\right) R_{3}\left(x_{2}, i_{2}\right) P_{3}\left(x_{3}, i_{3}\right) R_{3}\left(x_{4}, i_{4}\right)$. So $i_{1} \leq i_{2}=i_{3}<i_{4}, x_{k}=\left(x_{k, 1}, x_{k, 2}\right)$ for $k=2,3,4$ and $x_{4,1}=x_{4,2}$. If $x_{1} \in W$ then immediately we conclude $\left(x_{1}, i_{1}\right) P_{3}\left(x_{1}, i_{1}\right) R_{3}\left(x_{4}, i_{4}\right)$. If $x_{1}=\left(x_{1,1}, x_{1,2}\right)$ then $x_{1,1} R x_{2,1} P x_{3,1} R x_{4,1}=x_{4,2}$ so by property $(G R)$ exists a $x$ s.t. $x_{1,1} P x R x_{4,1}$ and so $\left(x_{1}, i_{1}\right) P_{3}\left(\left(x, x_{1,2}\right), i_{1}\right) R_{3}\left(x_{4}, i_{4}\right)$.

- given a $c s$-frame $(W, R, P)$ satisfying property $(G R)$ with initial family $I,\left(I, \Delta^{I}\right)$ is a globally right transitive reactive frame:

Let $\lambda w w^{\prime}, \lambda^{\prime} w^{\prime} w^{\prime \prime} \in \Delta^{I}$ then there are I-initial paths $\gamma s_{1} s_{2}$ and $\gamma^{\prime} s_{3} s_{4}\left(s_{i} \in W\right)$ s.t. $l_{I}\left(\gamma s_{1} s_{2}\right)=\lambda w w^{\prime}$ and $l_{I}\left(\gamma^{\prime} s_{3} s_{4}\right)=\lambda^{\prime} w^{\prime} w^{\prime \prime}$ (so $\left.s_{1} R s_{2} P s_{3} R s_{4}\right)$. By property $(G R)$ there exists $s \in W$ s.t. $s_{1} P s R s_{4}$. By initiality of $I$, there is some $\gamma^{\prime \prime}$ s.t. $\gamma^{\prime \prime} s$ is an I-initial path and so $\gamma^{\prime \prime} s s_{4}$, thus $l_{I}\left(\gamma^{\prime \prime} s s_{4}\right)=\lambda^{\prime \prime} w w^{\prime \prime} \in \Delta$. Applying lemma 3.7 we conclude that $\lambda^{\prime} w^{\prime} w^{\prime \prime} \sim \lambda^{\prime \prime} w w^{\prime \prime}$.

So

$$
L_{\Pi}^{4_{g r}}=\{\varphi: \varphi \text { is valid in every globally right transitive reactive frame }\} .
$$

Given $X \subsetneq \Pi, L_{X}^{4_{g r}}$ is not sound and complete for any class of reactive frames:

It follows from the proof in 3. for $L_{X}^{4_{r}}$. In the first case we can also conclude that the frame does not validate $\diamond_{R} \diamond_{P} \diamond_{R} p \rightarrow \diamond_{P} \diamond_{R} p$ thus $L_{X}^{4_{r l}} \subset L_{X}^{4_{r}}$ it is not sound in relation any class of frames containing a reactive frame with a path of the form $\lambda w_{0} w_{1} w_{2}$ with $w_{0} \neq w_{1}$. Neither it is complete with respect to the other two cases considered since both reactive frames used in the proof are also globally right transitive.

\subsection{Static and quasi-static}

Proposition 3.12 We have that $\left(\diamond_{P} \diamond_{R} p \rightarrow \diamond_{R} \diamond_{P} p\right) \leftrightarrow\left(\diamond_{R} \square_{P} p \rightarrow \square_{P} \diamond_{R} p\right) \in L_{X}$ and $L_{X}^{S}=L_{X} \oplus \diamond_{P} \diamond_{R} p \rightarrow \diamond_{R} \diamond_{P} p=L_{X} \oplus \diamond_{R} \square_{P} p \rightarrow \square_{P} \diamond_{R} p$ is sound and complete with respect to the class of $X$-reactive models over static reactive frames, i.e. satisfying:

$$
\lambda w, \lambda w^{\prime} \in \Delta \rightarrow \lambda w \sim \lambda^{\prime} w
$$


Notice that if $X=\Pi$, the new axioms are equivalent to $\diamond_{P} p \rightarrow p$ and that if we impose this axiom instead we get a result limited to this case. This tell us that in the other cases where we have this kind of restriction there may be better axiomatisations valid for all $X$.

Proof. Let $\operatorname{com}_{P R}^{r}=\diamond_{P} \diamond_{R} p \rightarrow \diamond_{R} \diamond_{P} p$ and $c h r_{P R}=\diamond_{R} \square_{P} p \rightarrow \square_{P} \diamond_{R} p$.

- Using the equality $L_{r, X}=L_{X}$ :

- chr $_{P R}$ implies $\operatorname{com}_{P R}^{r}$

$\mathcal{M}, \lambda w \vDash \diamond_{P} \diamond_{R} \varphi$, since $\varphi \rightarrow \square_{P} \diamond_{P} \varphi$ of $P$ we have $\mathcal{M}, \lambda w \models \diamond_{P} \diamond_{R} \square_{P} \diamond_{P} \varphi$. Applying $c h r_{P R}$ we get $\mathcal{M}, \lambda w \vDash \diamond_{P} \square_{P} \diamond_{R} \diamond_{P} \varphi$ and, again from $\psi \rightarrow$ $\square_{P} \diamond_{P} \psi$ (equivalent to $\diamond_{P} \square_{P} \psi \rightarrow \psi$ ), we obtain $\mathcal{M}, \lambda w \models \diamond_{R} \diamond_{P} \varphi$.

- $\operatorname{com}_{P R}^{r}$ implies chr $_{P R}$

$\mathcal{M}, \lambda w \vDash \diamond_{P} \square_{R} \varphi$, since $\varphi \rightarrow \square_{P} \diamond_{P} \varphi$, we have $\mathcal{M}, \lambda w \vDash \diamond_{P} \square_{R} \square_{P} \diamond_{P} \varphi$. Applying $\operatorname{com}_{P R}^{r}$ we get $\mathcal{M}, \lambda w \vDash \diamond_{P} \square_{P} \square_{R} \diamond_{P} \varphi$ and, again from $\diamond_{P} \square_{P} \psi \rightarrow$ $\psi$, we obtain $\mathcal{M}, \lambda w \vDash \square_{R} \diamond_{P} \varphi$.

- Soundness of $L_{X}^{S}$ comes easily from the soundness of the new axiom:

Given a $X$-reactive model $\mathcal{M}=(W, \Delta, v)$ over a static frame and $\lambda w \in \Delta$ s.t. $\mathcal{M}, \lambda w \vDash \diamond_{P} \diamond_{R} \varphi$ then there exists $\lambda^{\prime} w w^{\prime} \in \Delta$ s.t. $\mathcal{M}, \lambda^{\prime} w w^{\prime} \vDash \varphi$ thus $\lambda w w^{\prime} \in \Delta$ (since $\left.\lambda w \sim \lambda^{\prime} w\right)$ and $\mathcal{M}, \lambda w \vDash \diamond_{R} \diamond_{P} \varphi\left(\right.$ since $\left.t\left(\lambda w w^{\prime}\right)=t\left(\lambda^{\prime} w w^{\prime}\right)\right)$.

- For completeness we proceed as before:

- $\diamond_{P} \diamond_{R} p \rightarrow \diamond_{R} \diamond_{P} p$ is a Sahlqvist formula and so the canonical model $F=$ $(W, R, P)$ for $L_{X}^{S}$ satisfies $P R$-commutativity:

$$
\forall x y z \exists y^{\prime} x P y R z \rightarrow x R y^{\prime} P z
$$

since it is the first-order correspondent to the new axiom.

- PR-commutativity is preserved by $t_{1}$ :

Let $(x, i),(y, j),(z, k) \in W^{\prime}$ s.t. $(x, i) P^{\prime}(y, j) R^{\prime}(z, k)$. If $i=*$ then $(x, i)=$ $(y, j)$ and $(x, i) R^{\prime}(z, k) P^{\prime}(z, k)$. If $i \neq *$ then $x P y R z, i=j=\left(x^{\prime}, a\right)$ and $k=\left(z^{\prime}, b\right)$. So exists $y^{\prime}$ s.t. $x R y^{\prime} P z$, and so $t^{\prime}(x, v)$ is defined for any $v$ s.t. $v P z$. Or $i=j=k$, so $t^{\prime}(x, x)$ is defined and $(x, i) R^{\prime}\left(t^{\prime}(x, x), k\right) P^{\prime}(z, k)$, or $i=j \neq k$, so $t^{\prime}\left(x, z^{\prime}\right)$ is defined and $(x, i) R^{\prime}\left(t^{\prime}\left(x, z^{\prime}\right), k\right) P^{\prime}(z, k)$.

- Given a PR-commutative $c s$-frame with initial family $I$ then $\left(I, \Delta^{I}\right)$ is a static reactive frame:

Let $\lambda w, \lambda^{\prime} w \in \Delta^{I}$ so there is $\beta b_{1}$ and $\beta^{\prime} b_{1}^{\prime} I$-initial paths s.t. $l_{I}\left(\beta b_{1}\right)=\lambda w$ and $l_{I}\left(\beta^{\prime} b_{1}^{\prime}\right)=\lambda^{\prime} w$. Let us see that for every $\gamma=w_{1} \ldots w_{n}$ we have $\beta b_{1} \gamma$ iff exist $\gamma^{\prime}=v_{1} \ldots v_{n}$ s.t. $\beta^{\prime} b_{1} \gamma^{\prime}$ and $v_{i} P w_{i}$ for $i=1, \ldots, n$. By induction on $n$ (we do it only in one direction, the other is the same):

$-n=1$

$\beta b_{1} w_{1}$ is an I-initial path so by $P R$-commutativity exists $v_{1}$ s.t. $b_{2} R v_{1} P w_{1}$ and so $\beta^{\prime} b_{2} v_{1}$ is an $I$-initial path. 


$$
\begin{aligned}
& -n+1 \\
& \beta b_{1} w_{1} \ldots w_{n} w_{n+1} \text { is an I-initial path so by } P R \text {-commutativity exists } v_{n+1} \text { s.t. } \\
& v_{n} R v_{n+1} P w_{n+1} \text { and so } \beta^{\prime} b_{2} v_{1} \text { is an I-initial path. }
\end{aligned}
$$

Hence we conclude that $\lambda w \sim \lambda^{\prime} w$.

A static frame is a reactive frame that does not react, that is, the accessible worlds depend only on the current world and not on how you get there. It is clear that from such a reactive frame $(W, \Delta)$ we can obtain a classic Kripke frame $(W, R)$ where

$$
R=\{(w, v): w v \in \Delta\}=\{(w, v): \lambda w v \in \Delta\}
$$

It is straightforward to see that in such a reactive frame all the variants of transitivity and reflexivity on reactive frames coincide with the usual notions on the correspondent Kripke frame.

It is easy to see that a Kripke model over general frame $(W, R, P, A)$, where $P$ is a equivalence class, is a $\Pi$-shattered model iff $A \subseteq 2^{W}$ - the boolean algebra generated by the $P$ equivalence classes - is closed for the operators: $m_{R}(X)=\{x \in W: \exists y \in$ $W$ s.t. $x R y\}$ and $m_{P}(X)=\{x \in W: \exists y \in W$ s.t. $x P y\}$. Being $P$ an equivalence relation, $A$ is trivially closed under $m_{P}$. It is easy to see that $A$ being closed under $m_{R}$, means that if a world is in $m_{R}(X)$ so it must be all its $P$-class, which is corresponds to PRcommutativity. From the point of view of reactivity, the use of (shattered) general frames to deal with the restrictions over the valuations (even if only in the case of $X=$ $\Pi$ ) does not help, it demands a very strict interaction between $R$ and $P$, it corresponds, in the reactive level to ask it not to react! See [16] for an equivalent presentation of $\Pi$-shattered models (with only $\diamond_{R}$ ) and its relation with general frames.

Proposition 3.13 Let $L_{X}^{q S}=L_{X} \oplus \diamond_{R} \diamond_{P} p \rightarrow \diamond_{P} \diamond_{R} p$.

$L_{\Pi}^{q S}$ is sound and complete with respect to the class of (П-shattered models over) quasi-static reactive frames, i.e. that satisfy:

$$
\lambda w w^{\prime}, \lambda^{\prime} w^{\prime} \in \Delta \rightarrow \exists \lambda^{\prime \prime} \lambda^{\prime} w^{\prime} \sim \lambda^{\prime \prime} w w^{\prime} \in \Delta
$$

If $X \subsetneq \Pi, L_{X}^{q S}$ is not sound and complete with respect to (all X-models over) any class of reactive frames.

\section{Proof.}

- Soundness:

Given a $\Pi$-reactive model $\mathcal{M}=(W, \Delta, v)$ over a quasi-static reactive frame and $\lambda w \in \Delta$ s.t. $\mathcal{M}, \lambda w \vDash \diamond_{R} \diamond_{P} \varphi$ then there exists $\lambda w w^{\prime}, \lambda^{\prime} w^{\prime} \in \Delta$ s.t. $\mathcal{M}, \lambda^{\prime} w^{\prime} \vDash \varphi$ thus $\lambda^{\prime} w^{\prime} \sim \lambda^{\prime \prime} w w^{\prime} \in \Delta$ and $\mathcal{M}, \lambda^{\prime \prime} w w^{\prime} \vDash \varphi$ (by lemma 2.5). Hence $\mathcal{M}, \lambda w \vDash$ $\diamond_{P} \diamond_{R} \varphi\left(\right.$ since $\left.t\left(\lambda w w^{\prime}\right)=t\left(\lambda^{\prime} w w^{\prime}\right)\right)$.

- Completeness: 
$\diamond_{R} \diamond_{P} p \rightarrow \diamond_{P} \diamond_{R} p$ is a Sahlqvist formula and so the canonical frame $F=$ $(W, R, P)$ for $L_{X}^{q S}$ satisfies $R P$-commutativity:

$$
\forall x y z \exists y^{\prime} x R y P z \rightarrow x P y^{\prime} R z
$$

since it is the first-order correspondent to the new axiom.

Given $M=(W, R, P, V)$ a $R P$-commutative shattered frame, let $M^{\prime}=$ $\left(W^{\prime}, R^{\prime}, P^{\prime}, V^{\prime}\right)$ be defined by:

- $W^{\prime}=\left(W^{+}\right)^{3} \cup\left(W^{+}\right)^{2} \times\{*\}$

- $(a, b, c) R^{\prime}\left(a^{\prime}, b^{\prime}, c^{\prime}\right)$ iff $c=* \& a=a^{\prime}$ or $c \neq * \neq c^{\prime} \& o(a) \operatorname{Ro}\left(a^{\prime}\right) \&$ $\left(a \in W \& a^{\prime}=b^{\prime}=c^{\prime}\right.$ or $a \notin W \&$ $\left(b^{\prime} \neq a^{\prime} \neq c^{\prime} \& r(a)=a^{\prime}\right.$ or $r(a)=b^{\prime} \& a^{\prime}=c^{\prime}$ or $\left.\left.r(a)=c^{\prime} \& a^{\prime}=b^{\prime}\right)\right)$

- $(a, b, c) P^{\prime}\left(a^{\prime}, b^{\prime}, c^{\prime}\right)$ iff $(b, c)=\left(b^{\prime}, c^{\prime}\right) \& o(a) P o\left(a^{\prime}\right)$

- $(a, b, c) \in V^{\prime}(p)$ iff $o(a) \in V(p)$,

where $o\left(w_{1} \ldots w_{n}\right)=w_{1}, t\left(w_{1} \ldots w_{n}\right)=w_{n}$ and $r\left(w_{1} \ldots w_{n}\right)=w_{2} \ldots w_{n}$.

We have that $\left(W^{\prime}, R^{\prime}, P^{\prime}\right)$ is a $R P$-commutative cs-frame and the $M^{\prime}$ is a bounded morphic image of $M$ :

- $\left(W^{\prime}, R^{\prime}, P^{\prime}\right)$ is a $R P$-commutative Let $(a, b, c) R^{\prime}\left(a^{\prime}, b^{\prime}, c^{\prime}\right) P^{\prime}\left(a^{\prime \prime}, b^{\prime \prime}, c^{\prime \prime}\right)$. If $c=*$ then $a=a^{\prime}$ and $o(a)=o\left(a^{\prime}\right) P o\left(a^{\prime \prime}\right)$ thus

$$
(a, b, c) P^{\prime}\left(a^{\prime \prime}, b, c\right) R^{\prime}\left(a^{\prime \prime}, b^{\prime \prime}, c^{\prime \prime}\right) \text {. }
$$

If $c \neq * \neq c^{\prime}$ then $o(a) \operatorname{Ro}\left(a^{\prime}\right) \operatorname{Po}\left(a^{\prime \prime}\right)$ so exists $w$ s.t. $o(a) P w R o\left(a^{\prime \prime}\right)$. We have three possibilities:

or $b^{\prime}=b^{\prime \prime} \neq a^{\prime \prime} \neq c^{\prime \prime}=c^{\prime}$ and so $(a, b, c) P^{\prime}\left(w a^{\prime \prime}, b, c\right) R^{\prime}\left(a^{\prime \prime}, b^{\prime \prime}, c^{\prime \prime}\right)$;

or $a^{\prime \prime}=b^{\prime \prime}$ thus

$$
(a, b, c) P^{\prime}\left(w c^{\prime \prime}, b, c\right) R^{\prime}\left(a^{\prime \prime}, b^{\prime \prime}, c^{\prime \prime}\right)
$$

or $a^{\prime \prime}=c^{\prime \prime}$ and

$$
(a, b, c) P^{\prime}\left(w b^{\prime \prime}, b, c\right) R^{\prime}\left(a^{\prime \prime}, b^{\prime \prime}, c^{\prime \prime}\right)
$$

- Clearly $V^{\prime}$ is admissible and that any choice of I picking one element from each $P$-class and containing $\left\{(a, a, *): a \in W^{+}\right\}$works as an initial family.

- Let us check that it is coherent. Let $(a, b, c) R^{\prime}\left(a^{\prime}, b^{\prime}, c^{\prime}\right),(a, b, c) R^{\prime}\left(a^{\prime \prime}, b^{\prime \prime}, c^{\prime \prime}\right)$ and $\left(a^{\prime}, b^{\prime}, c^{\prime}\right) P^{\prime}\left(a^{\prime \prime}, b^{\prime \prime}, c^{\prime \prime}\right)$. From $\left(a^{\prime}, b^{\prime}, c^{\prime}\right) P^{\prime}\left(a^{\prime \prime}, b^{\prime \prime}, c^{\prime \prime}\right)$ we conclude that $\left(b^{\prime}, c^{\prime}\right)=\left(b^{\prime \prime}, c^{\prime \prime}\right)$. If $c=*$ then $a^{\prime}=a=a^{\prime \prime}$. If $c \neq * \neq c^{\prime}$, then or $a \in W$ and $a^{\prime}=b^{\prime}=c^{\prime}=c^{\prime \prime}=b^{\prime \prime}=a^{\prime \prime}$; or $a \notin W$, in which case we have three subcases: or $b^{\prime} \neq a^{\prime} \neq c^{\prime}$ and so $r(a)=a^{\prime}=a^{\prime \prime}$; or $a^{\prime}=b^{\prime}$, thus $r(a)=c^{\prime}=c^{\prime \prime}$ and $a^{\prime \prime}=b^{\prime \prime}=b^{\prime}=a^{\prime}$; or $a^{\prime}=c^{\prime}$ and $r(a)=b^{\prime}=b^{\prime \prime}$ so $a^{\prime \prime}=c^{\prime \prime}=c^{\prime}=a^{\prime}$. In any case, $\left(a^{\prime}, b^{\prime}, c^{\prime}\right)=\left(a^{\prime \prime}, b^{\prime \prime}, c^{\prime \prime}\right)$. 
- Let $f:\left(W^{+}\right)^{3} \rightarrow W$ be defined by $f((a, b, c))=o(a)$.

It is straightforward to see that $f$ is surjective, let us see that it is actually a p-morphism:

The condition in the valuation is trivial as before;

* $f$ is a p-morphism in $R$

- $(a, b, c) R^{\prime}\left(a^{\prime}, b^{\prime}, c^{\prime}\right)$ then $f((a, b, c))=o(a) \operatorname{Ro}\left(a^{\prime}\right)=f\left(\left(a^{\prime}, b^{\prime}, c^{\prime}\right)\right)$,

- $f((a, b, c)) R w$,

if $a \in W$ then $(a, b, c) R^{\prime}(w, w, w)$ otherwise $(a, b, c) R^{\prime}(w, r(a), w)$;

* $f$ is a p-morphism in $P$

- $(a, b, c) P^{\prime}\left(a^{\prime}, b^{\prime}, c^{\prime}\right)$ then $f((a, b, c))=o(a) P o\left(a^{\prime}\right)=f\left(\left(a^{\prime}, b^{\prime}, c^{\prime}\right)\right)$,

- $f((a, b, c)) P w$ then $(a, b, c) P^{\prime}(w, b, c)$;

- Given a PR-commutative $c s$-frame with initial family $I$ then $\left(I, \Delta^{I}\right)$ is a such a reactive frame:

Let $\lambda w w^{\prime}, \lambda^{\prime} w^{\prime} \in \Delta^{I}$, so there is $\gamma, \gamma^{\prime} \in W^{*}$ and $s, s^{\prime}, s^{\prime \prime} \in W$ s.t. $\gamma s s^{\prime}$ and $\gamma^{\prime} s^{\prime \prime}$ are $I$-initial paths, $l_{I}\left(\gamma s s^{\prime}\right)=\lambda w w^{\prime}$ and $l_{I}\left(\gamma^{\prime} s^{\prime \prime}\right)=\lambda^{\prime} w^{\prime}$. So $s R s^{\prime} P s^{\prime \prime}$, thus there is $t$ s.t. $s P t R s^{\prime \prime}$. Let $\gamma^{\prime \prime}$ be s.t. $\gamma^{\prime \prime} t$ is an I-initial path, $\gamma^{\prime \prime} t s^{\prime \prime}$ is I-initial too. Furthermore, using lemma 3.7, we conclude that $\lambda w^{\prime} \sim \lambda^{\prime \prime} w w^{\prime}=l_{I}\left(\gamma^{\prime \prime} t s^{\prime \prime}\right)$.

It is clear that if a reactive frame is static then it is also quasi-static. Furthermore it is interesting to notice that if a frame is static all the variants of transitivity and reflexivity coincide.

\subsection{Open problem - symmetry}

Our method seems not to be as fruitful with the notions of generalized symmetry. We are unable to prove reactive frame completeness. To prove that it is sound to a certain class of reactive frames and that it is complete to the correspondent class of shattered frames is straightforward as before. However completeness cannot be proved in the same way. We just cannot simply pass from the shattered to cs-frames. Let us look just to the case of strong symmetry.

Conjecture 3.14 Let $L_{X}^{B}=L_{X} \oplus p \rightarrow \square_{R} \diamond_{R} p$. $L_{\Pi}^{B}$ is sound and complete with respect to the class of reactive frames that are strongly symmetric, i.e. satisfying:

$$
\lambda w w^{\prime} \in \Delta \rightarrow \lambda w \sim \lambda w w^{\prime} w \in \Delta
$$

Still we have not been able to prove it is not complete either. We present the positive result as a conjecture and add a brief explanation on how our attempts failed.

We present a $R$-reflexive shattered frame that cannot be transformed into a cs-frame using the classic backward truth preserving transformations.

If a shattered frame $(W, R, P)$ with $R \neq \emptyset$ satisfies for all $w$ there is $w^{\prime}$ s.t. $w P w^{\prime}$, there is no $w^{\prime \prime}$ satisfying $w^{\prime} R w^{\prime \prime}$ or $w^{\prime \prime} R w^{\prime}$, then it does not admit an initial family. 
Let us assume the contrary, let I be its initial family and

$$
A=\left\{w: \text { there is no } w^{\prime} \text { s.t. } w R w^{\prime} \text { or } w^{\prime} R w\right\} .
$$

Since $I R$-generates $W$, the isolated points must be in $I$, so $A \subseteq I$. From the fact that $I$ picks only one element from each $P$-class and for every $w$ there is $w^{\prime} \in A$ s.t. $w P w^{\prime}$ we have that $I=A$. As there is an element in $W-A I$ does not $R$-generate all $W$.

It is also clear that a frame that has such a frame as generated subframe cannot have an initial family. Or else the elements of the initial family of the bigger frame, present in the smaller one, would be an initial family to the latest.

Furthermore, the operation of taking pre-images, ultrafilter extensions and ultrapowers preserves this (bad) property.

The following shattered frame $\left(\{0,1\},\{(1,1)\},\{0,1\}^{2}\right)$ is $R$-symmetric and satisfies the bad property. Hence classical ways of generating new models from old preserving modal satisfaction do not allow us to find a general recipe to convert the relevant shattered frames into the correspondent cs-frames. In particular no variation of blow up will work.

This may not be a limitation on the method, instead it may be that the conjecture is not true and that the missing axiom would restrict us to a class of shattered frames that do not have this property. We have not been able to prove either way.

In the presence of (strong) reflexivity this problem disappears and the blow up method works:

Proposition 3.15 Let $L_{X}^{T B}=L_{X}^{T} \oplus p \rightarrow \square_{R} \diamond_{R} p=L_{X}^{B} \oplus p \rightarrow \diamond_{R} p$. $L_{\Pi}^{T B}$ is sound and complete with respect to the class of strongly symmetric reactive frames that are also strongly reflexive.

Proof. Soundness is just as before obtained by checking that $p \rightarrow \square_{R} \diamond_{R} p$ is sound:

Given a strongly symmetric reactive model $\mathcal{M}=(W, \Delta, v)$ and $\lambda w \in \Delta$ s.t. $\mathcal{M}, \lambda w \models$ $\varphi$ then given $v$ s.t. $\lambda w v \in \Delta$ then $\lambda w v \sim \lambda w v w \in \Delta$ so, by proposition $2.5, \mathcal{M}, \lambda w v w \vDash \varphi$ thus $\mathcal{M}, \lambda w v \vDash \diamond_{R} \varphi$. Hence and $\mathcal{M}, \lambda w \models \square_{R} \diamond_{R} \varphi$

In this case we are able to prove completeness by applying the blow up method:

Given $M=(W, R, P, V)$ a $R$-symmetric and $R$-reflexive shattered frame, let $M^{\prime}=$ $\left(W^{\prime}, R^{\prime}, P^{\prime}, V\right)$ be defined by:

- $W^{\prime}=\bigcup_{n<\omega} W^{2 n+1} \times \bigcup_{n<\omega} W^{2 n+1}$

- $(x, y) R^{\prime}\left(x^{\prime}, y^{\prime}\right)$ iff $x=x^{\prime}$ or

$\left(y=y^{\prime} \& w R w^{\prime} \&\left(x=z \& x^{\prime}=z^{\prime}\right.\right.$ or $\left.x^{\prime}=z \& x=z^{\prime}\right) \&$

$\left.z=(w, \bar{v}) \& z^{\prime}=\left(w^{\prime}, w^{\prime}, w, \bar{v}\right)\right)$

- $(x, y) P^{\prime}\left(x^{\prime}, y^{\prime}\right)$ iff $y=y^{\prime} \& x=(w, \bar{v}) \& x^{\prime}=\left(w^{\prime}, \bar{v}\right) \& w P w^{\prime}$

- $\left(\left(x_{1}, \ldots, x_{k}\right), w\right) \in V^{\prime}(p)$ iff $x_{1} \in V(p)$.

where $\bar{v}=v_{1}, \ldots, v_{k}$.

We have that $\left(W^{\prime}, R^{\prime}, P^{\prime}\right)$ is a cs-frame (where $R^{\prime}$ is clearly symmetric and reflexive) and the $M^{\prime}$ is a bounded morphic image of $M$ : 
- clearly $V^{\prime}$ is admissible and that any choice of I picking one element from each $P$-class and containing $\left\{(x, y) \in W^{\prime}: x=y\right\}$ works as an initial family.

- let us check that it is coherent. Given $(x, y),\left(x^{\prime}, y^{\prime}\right),\left(x^{\prime \prime}, y^{\prime \prime}\right) \in W^{\prime}$. If $\left(x^{\prime}, y^{\prime}\right) P^{\prime}$ $\left(x^{\prime \prime}, y^{\prime \prime}\right)$ then $x^{\prime}=(v, \bar{v}), x^{\prime \prime}=\left(v^{\prime}, \bar{v}\right)\left(\right.$ so $\left.\left|x^{\prime}\right|=\left|x^{\prime \prime}\right|\right)$ with $w P w^{\prime}$ and $y^{\prime}=y^{\prime \prime}$. Let us assume that we also have $(x, y) R^{\prime}\left(x^{\prime}, y^{\prime}\right)$ and $(x, y) R^{\prime}\left(x^{\prime \prime}, y^{\prime \prime}\right)$.

If $x=x^{\prime}$ and $x \neq x^{\prime \prime}$ then $\left|x^{\prime}\right|=|x| \neq\left|x^{\prime \prime}\right|$ which contradicts $\left(x^{\prime}, y^{\prime}\right) P^{\prime}\left(x^{\prime \prime}, y^{\prime \prime}\right)$, so $x=x^{\prime \prime}$. The same applies if $x=x^{\prime \prime}$ and $x \neq x^{\prime}$.

If $x \neq x^{\prime}$ and $x^{\prime} \neq x^{\prime \prime}$ then or $x=(w, \bar{v})$ and $x^{\prime}=\left(w^{\prime}, w^{\prime}, w, \bar{v}\right)=x^{\prime \prime}$, or $x=\left(w^{\prime}, w^{\prime}, w, \bar{v}\right)$ and $x^{\prime}=(w, \bar{v})=x^{\prime \prime}$.

In any case: $\left(x^{\prime}, y^{\prime}\right)=\left(x^{\prime \prime}, y^{\prime \prime}\right)$.

- let $f: W^{\prime} \rightarrow W$ be defined by $f(((w, \bar{x}), y))=w$.

It is straightforward to see that $f$ is surjective, let us see that it is actually a p-morphism:

The condition in the valuation is trivial as before;

- $f$ is a p-morphism in $R$

* $((w, \bar{x}), y) R^{\prime}\left(\left(w^{\prime}, \bar{x}\right), y^{\prime}\right)$ then or $w=w^{\prime}$ or $w R w^{\prime}$, in any case $f\left(\left(\left(w^{\prime}, \bar{x}\right), y\right)\right)=$ $w R w^{\prime}=f\left(\left(\left(w^{\prime}, \bar{x}\right), y^{\prime}\right)\right)$.

* $f(((w, \bar{x}), v)) R w^{\prime}$ then $(((w, \bar{x}), v)) R^{\prime}\left(\left(\left(w^{\prime}, w^{\prime}, w, \bar{x}\right), v\right)\right)$;

- $f$ is a p-morphism in $P$

* $((w, \bar{x}), y) P^{\prime}\left(\left(w^{\prime}, \bar{x}\right), y^{\prime}\right)$ then $f\left(\left(\left(w^{\prime}, \bar{x}\right), y\right)\right)=w P w^{\prime}=f\left(\left(\left(w^{\prime}, \bar{x}\right), y^{\prime}\right)\right)$.

* $f(((w, \bar{x}), v)) P w^{\prime}$ then $(((w, \bar{x}), v)) P^{\prime}\left(\left(\left(w^{\prime}, w, \bar{x}\right), v\right)\right)$.

In proposition 3.8 we have checked that given a $R$-reflexive $c s$-frame with initial family $I$ then $\left(I, \Delta^{I}\right)$ is a strongly reflexive reactive frame. Let us see now that if the cs-frame is also $R$-symmetric then $\left(I, \Delta^{I}\right)$ is also strongly symmetric:

Let $\lambda w w^{\prime} \in \Delta^{I}$, so there is $\gamma \in W^{*}$ and $s, s^{\prime} \in W$ s.t. $\gamma s s^{\prime}$ is an I-initial path and $l_{I}\left(\gamma s s^{\prime}\right)=\lambda w w^{\prime}$. Since $F$ is $R$-symmetric we have that $\gamma s s^{\prime} s$ is also an $I$-initial path and clearly $l_{I}\left(\gamma s s^{\prime} s\right)=\lambda w w^{\prime} w \in \Delta$. Applying lemma $3.7 w e$ conclude that $\lambda w \sim \lambda w w^{\prime} w$.

This result tells us that if the above conjecture is not true, the formula valid in all strong symmetric reactive frames that is not in $L_{X}^{B}$ must be a $L_{X}$-consequence of $p \rightarrow \diamond_{R} p$.

\section{Results and final comments}

Table 1 summarizes the main results presented in this paper. One can read along its lines the correspondence between logic, reactive frame property (including its dependence on $X$ ) and the shattered frame unfolded property. 
For example, in the first line we see that the logic $L_{X}$ corresponds to all shattered frames and to the whole class of ( $X$-models over) reactive frames, hence being the smallest (minimal) "reactive" logic. In general there is a dependence on $X$, for instance in the sixth line, we see that the completeness of $L_{X}^{T}=L \oplus p \rightarrow \diamond p$ with respect to the class of (all $X$-models over) strong reflexive reactive frames requires that $X=\Pi$, i.e. that all variables have to be fixed. We do not know if this dependence can be avoided by a more inspired choice of axiom as in the case of static reactive frames, see proposition 3.12 .

In section 3.3 we stated that the question of axiomatizing the generalized notions of symmetry is still open. The same happens with many other completeness results. It is not clear that the combination of the axioms corresponding to certain reactive properties, will correspond to the combination of those properties. This is the case in the classical setting when we add reflexivity to transitivity or symmetry. It may be that the reactive properties interact and those classes satisfy formulas not captured by the axioms. It certainly does not follow from the results we presented above. For that to happen the (blow up) transformation would have to preserve the combination of all properties in question. The fact is that we have been unable to find a transformation that would work for all cases, in particular when considering generalizations of different properties. In this sense our choice of transformations is very weak. By contrast, in the strong symmetry plus strong reflexivity case we see how gathering properties may also be helpful. The strong symmetry case alone is worse behavioured that the combined one.

In this paper we have used classical tools to study completeness on a new interpretation of modal logic. We have been successful in studying some of its subsystems although we are aware that it may fail (see section 3.3) and we should not expect it to work in every case. Furthermore it would be interesting to find some new methods that would allow a more direct way of studying these logics, leading to a reactivisation of modal techniques. 


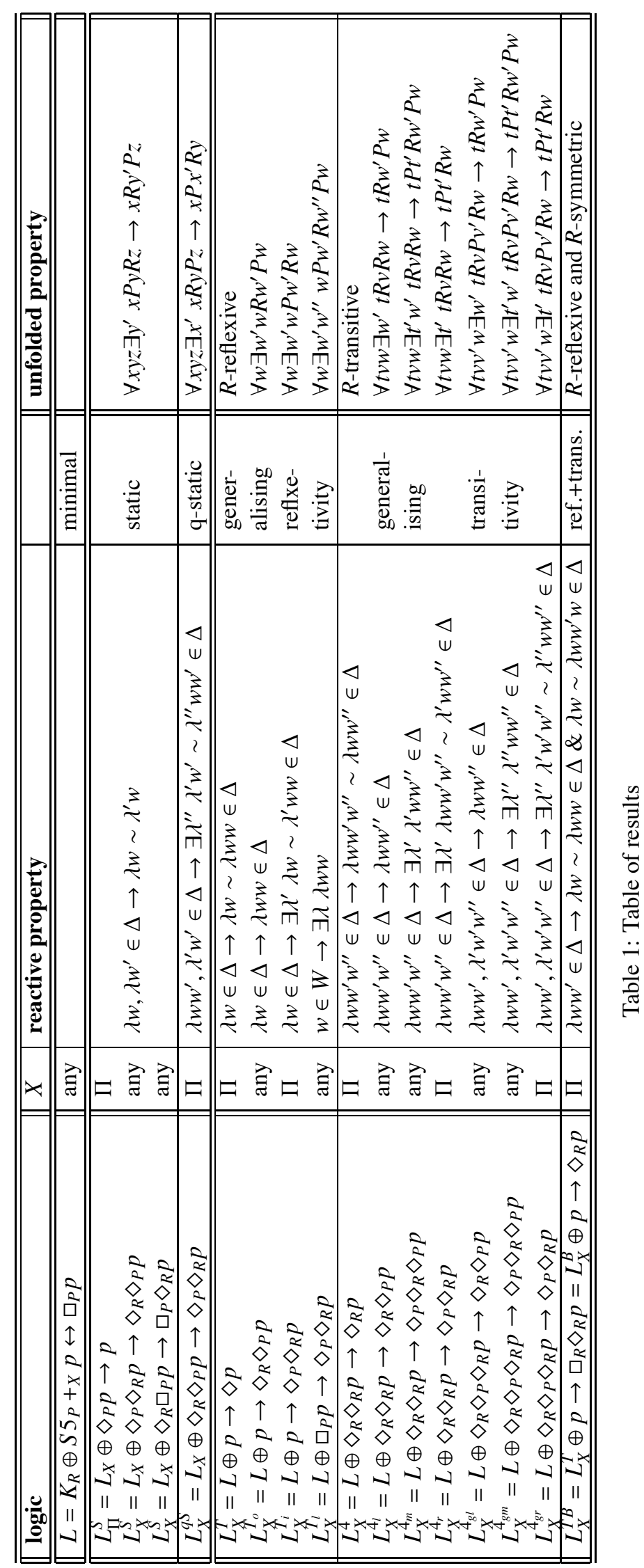




\section{References}

[1] D. Gabbay, H. Barringer and J. Woods. Temporal Dynamics of Argumentation Networks. In Volume Dedicated to Joerg Siekmann, D. Hutter and W. Stephan, editors. Mechanising Mathematical Reasoning, Springer Lecture Notes in Computer Science 2605, pp. 59-98, 2005.

[2] D. M. Gabbay Reactive Kripke Semantics and Arc Accessibility In Pillars of Computer Science*: Essays Dedicated to Boris (Boaz) Trakhtenbrot on the Occasion of His 85th Birthday, Arnon Avron, Nachum Dershowitz, and Alexander Rabinovich, editors, Lecture Notes in Computer Science, vol. 4800, SpringerVerlag, Berlin, 2008 pp 292-341

[3] D. M. Gabbay, H. Barringer and D. Rydeheard. Reactive Grammars. to appear in a LNCS volume in Honour of Yakov Choueka, Springer .N Dershovitz editor

[4] D. M. Gabbay and M. Crochemore. Reactive Automata. Submitted to Information and Computation

[5] D. M. Gabbay and S. Marcelino. Switch Graphs, in preparation.

[6] D. M. Gabbay. Reactive Kripke Models and Contrary-to-duty Obligations. In DEON -2008: Deontic Logic in computer science, Ron van der Meyden and Leendert van der Torre, eds. LNAI 5076, pp 155-173, Springer 2008

[7] D. M. Gabbay and K. Schlechta. A Theory of Hierarchical Conditionals. To appear in Journal of Logic Language and Information, DOI 10.1007/s10849-0099096-7.

[8] D. M. Gabbay. Reactive Kripke Models and Contrary-to-duty Obligations. EXpanded version, to appear in Journal of Applied Logic.

[9] D. M. Gabbay and K. Schlechta. Reactive Preferential Structures and Nonmonotonic consequence. In Review of Symbolic Logic, Vol 2 No 2 ,2009, pp 414-450, CUP CUP, RSL 08-048.

[10] D. M. Gabbay and K. Schlechta. Cumulativity Without Closure of the Domain under Finite Unions. The Review of Symbolic Logic , 1, 3:372-392, 2008.

[11] D. M. Gabbay and K. Schlechta. An Analysis of Defeasible Inheritance Systems. Logic Journal of the IGPL, 2009, pp 17-54.

[12] D. M. Gabbay and K. Schlechta. Size and Logic. To appear in Review of Symbolic Logic, CUP.

[13] D. M. Gabbay and K. Schlechta. Reactive description logics.

[14] R. Goldblatt. 1987 Logics of Time and Computation. Center for the Study of Language and Information. 
[15] V. Goranko. Completeness and Incompleteness in the Bimodal Base $L(R,-R)$. In Mathematical Logic, P. Petkov (ed.) Plenum Press, New York, 1990, pp. 311-327.

[16] Alexander Kurz, Sequence Frames, Proc. Verif. in New Orientation, Univ. Maribor (1995)

http://www.cs.le.ac.uk/people/akurz/LMU/maribor.ps.gz

[17] P. Blackburn, M. de Rijke, Y. Venema, Modal Logic, Cambridge: Cambridge University Press, 2001. 